\title{
MiR-124 and small molecules synergistically regulate the direct generation of neuronal cells from rat cortical reactive astrocytes
}

\section{Yangyang Zheng}

Jilin University https://orcid.org/0000-0002-8249-0554

\section{Zhehao Huang}

China-Japan Union Hospital of Jilin University

\section{Jinying Xu}

Jilin University

\section{Kun Hou}

Jilin University First Hospital

\section{Yifei Yu}

Jilin University

\section{Shuang LV}

Jilin University

Lin Chen

Jilin University

Yulin Li

Jilin University

Chengshi Quan

Jilin University

Guangfan Chi ( $\sim$ guangfan130@jlu.edu.cn )

\section{Research}

Keywords: central nervous system injury, glial scar, miR-124, small molecules, reactive astrocytes, neuron reprogramming; neuronal regeneration

Posted Date: August 25th, 2020

DOI: https://doi.org/10.21203/rs.3.rs-61060/v1

License: (c) (i) This work is licensed under a Creative Commons Attribution 4.0 International License.

Read Full License 


\section{Abstract}

Background冈|rreversible neuron loss caused by central nervous system injuries usually lead to persistent neurological dysfunction. Reactive astrocytes, because of their high proliferative capacity, proximity to neuronal lineage, and significant involvement in glial scarring, are ideal starting cells for neuronal regeneration. Having previously identified several small molecules as important regulators of astrocyteto-neuron reprogramming, our aim in this study was to explore whether other small molecules and miR124, a key neural differentiation mediator, could co-regulate reactive astrocyte-to-neuron conversion.

Methods: MiR-124, ruxolitinib, SB203580, and forskolin were used to induce postnatal rat cortex reactive astrocytes, and the neuronal phenotype of the induced cells was characterised. To understand the genetic changes, RNA-sequencing analyses were performed on reactive astrocytes, induced neurons, and rat neurons, and the mechanisms underlying the regulatory role of miR-124 during the neuronal conversion was explored.

Results囚MiR-124, ruxolitinib, SB203580, and forskolin could co-convert rat cortical reactive astrocytes into neurons. The induced cells had reduced astroglial properties, displayed typical neuronal morphologies, and expressed neuronal markers, reflecting $25.9 \%$ of cholinergic neurons. Gene analysis revealed that induced neuron gene expression patterns were more similar to that of primary neurons than of initial reactive astrocytes. On the molecular level, miR-124-driven neuronal differentiation of reactive astrocytes was via targeting of the SOX9-NFIA-HES1 axis to inhibit HES1 expression.

Conclusions $₫$ Providing a novel approach for inducing endogenous rat cortical reactive astrocytes into neurons by co-regulation involving miR-124 and three small molecules, our research has potential implications for inhibiting glial scar formation and promoting neuronal regeneration after central nervous system injury or disease.

\section{Background}

Functional neuron loss caused by central nervous system (CNS) injuries or diseases is usually irreversible and destructive [1, 2]. Upon injury stimulation, glial cells, especially astrocytes, proliferate and migrate to become reactive astrocytes (RAs) [3], eventually forming glial scars that impair neuronal regeneration [4]. Since the regenerative capacity of the CNS is limited [5], it seems meaningful to eliminate the neurosuppressive effects of glial scars and restore neural function. Fortunately, cell reprogramming using somatic cells to produce functional neurons addresses these issues [6]. Astrocytes, the predominant glial cells in the mammalian CNS, play vital roles in mediating neurotransmitter homeostasis and synaptic plasticity $[7,8]$. Like neurons, they originate from neural stem cells (NSCs), and blocking astrocytic signals and activating neurophenotypic signals can promote transitions of astrocyte-to-neuron states without lineage switching [9]. As autogenous cells and main components of glial scars, astrocytes eliminate the possibility of immunorejection; furthermore, neuronal conversion enables valuable new 
local neural circuit formation. Thus, astrocytes are the most promising candidates for neuronal reprogramming.

Transcription factors (TFs) such as NEUROG2, ASCL1, and DLX2 can directly reprogram astrocytes into neurons [10]. However, ectopic TF expression by viruses and the difficulties in delivering them into the CNS hinder their applications [11]. Alternatively, the biological effects of small molecules are finetuneable and reversible; thus, their development into pharmaceuticals can provide broad application prospects for neuronal reprogramming without genetic modification [12]. The small molecule cocktail of VPA, CHIR99021, RepSox, forskolin, i-BET151, and ISX-9 reprogrammed adult astrocytes into mainly glutamatergic neurons [13], and foetal astrocyte-derived functional neurons could be obtained via mediation by a small molecule cocktail of SB431542, LDN193189, CHIR99021, and 2,4-diamino-5phenylthiazole (DAPT) [14]. However, since the signals activated or inhibited by each small molecule differ, inconsistent chemical combinations lead to variable neuronal properties and conversion efficiencies. Additionally, the glutamatergic neurons produced in previous studies could not fully meet the needs of CNS injuries or diseases of other subtypes. Therefore, simplifying chemical compositions, improving conversion efficiency, inducing other subtype-specific neurons, and exploring more regulatory signals are critical for astrocyte-to-neuron reprogramming.

Combining small molecules with other agents to synergistically improve reprogramming effects has also garnered much attention. MicroRNAs (miRs) are small non-coding ribonucleic acids (RNAs) (20-22 nucleotides) that bind to the 3' untranslated region of target messenger RNAs) (mRNAs) to trigger translational repression or degradation [15] and can mediate direct neuronal reprogramming in vitro and in vivo $[16,17]$. MiR-124, one of the most abundant characteristic miRs in adult mammalian brains [18], is predominantly expressed in neurons, and its expression gradually increases with neuronal maturation [19]. Moreover, miR-124 is a neuronal fate determinant for the subventricular zone, which mediates neurogenesis in adult mammalian brains. MiR-124 overexpression can induce neuron-specific differentiation and govern the dendritic plasticity of NSCs [20,21]; in an ischaemic injury model, miR-124 administration could promote cortical neurogenesis [22]. Additionally, fibroblasts or HeLa cells with increasing miR-124 levels showed an expression profile shift toward that of a neuronal phenotype [23, 24]. This suggests that miR-124 not only regulates physiological and pathological neuronal differentiation of NSCs, but also enables some non-nervous system cells to acquire neuronal identities. However, whether miR-124 has similar neuromodulative effects on RAs has not yet been assessed. Repressing the RNA-binding protein, PTBP1 has recently been reported to mediate astrocyte-todopaminergic neuron conversion in a Parkinson's disease (PD) mouse model $[25,26]$. We suspect that as one of the targets of miR-124 [27], Ptbp1 of RAs may be targeted by miR-124 to promote their neuronal differentiation.

Here we present a novel RA-to-neuron reprogramming strategy using miR-124, ruxolitinib, SB203580, and forskolin, a combination that inhibited RA glial scar formation ability and also promoted neuronal regeneration. Among the small molecules, ruxolitinib and SB203580, inhibitors of the JAK/STAT and P38 MAPK pathways, respectively, involved in astrogliosis, have not been used for neuronal reprogramming 
before. Considering that miR-124 drives neuronal differentiation by inhibiting NOTCH signalling via the SOX9-NFIA-HES1 axis, we verified that simultaneous modulation of NOTCH, JAK/STAT, and P38 MAPK signals is sufficient to convert rat cortex RAs into mainly cholinergic neurons in vitro, opening new avenues for developing future CNS repair therapies.

\section{Methods}

\section{Isolation and culture of primary cortical RAs}

Primary cortical astrocytes were isolated from neonatal Wistar rats ( $1-3$ days old). Briefly, after removal of the blood vessels and pia mater, cerebral cortices were dissected and digested using papain (100 units, $40.4 \mathrm{mg} / \mathrm{ml}$; Worthington, LS003127), L-cysteine (0.0036-0.0042 g/ml), and DNAse I (4 mg/ml; Worthington, LS002006) at $34^{\circ} \mathrm{C}$; after $45 \mathrm{~min}$, the reaction was terminated using $20 \%$ foetal bovine serum (FBS) (Sigma) and 10X Earle's balanced salt solution (Sigma, E7510). Cells were passed through a $40 \mu \mathrm{m}$ filter (Greiner, 542040) to obtain a single-cell suspension and plated at a density of $20 \times 10^{5}$ cells/T25 flask in RA conditioned medium (AM) (Dulbecco's Modified Eagle's medium/F12 [DMEM/F12] [Corning, 10-0920CVR] containing 10\% FBS, 1X N2 [Gibco, 17502-048], 5 ng/ml bFGF [PeproTech, 400-2950], $10 \mathrm{ng} / \mathrm{ml}$ TGF- $\beta 1$ [PeproTech, 96-100-21-10], and 1\% penicillin-streptomycin [Invitrogen, 15140122]) in a $5 \% \mathrm{CO}_{2}$ humidified incubator at $37^{\circ} \mathrm{C}$. Culture medium was refreshed once every 2 days. About 1 week later, when cultured cells had reached $90 \%$ confluence, flasks were moved to a shaker at $37^{\circ} \mathrm{C}(280$ $\mathrm{rpm} / \mathrm{min}, 10 \mathrm{~h} /$ day) for 2 days. Thereafter, $20 \mu \mathrm{M}$ cytarabine (Sigma, C1768) was added for $24 \mathrm{~h}$ to obtain high purity RAs. Subsequent experiments were performed when GFAP-positive cells exceeded 95\% of the total cell number.

\section{Isolation and culture of primary rat cortical neurons}

Single-cell suspensions of neonatal Wistar rat (1-3 days old) cortices were obtained by the method described for primary cortical astrocyte isolation. Subsequently, cells were plated (at a density of $20 \times 10^{5}$ cells/poly-D-lysine hydrobromide [Sigma, P6407] pre-coated T25 flask) in neuron conditioned medium (NM) (Neurobasal [Gibco, 10888022] supplemented with 5\% FBS, 1\% B27 (Invitrogen, 17504044), 10 $\mathrm{ng} / \mathrm{ml} \mathrm{BDNF}, 15 \mathrm{ng} / \mathrm{ml}$ GDNF, $1 \%$ L-glutamine [Gibco, 25030081], and 1\% penicillin-streptomycin) in a $5 \%$ $\mathrm{CO}_{2}$ humidified incubator at $37^{\circ} \mathrm{C}$. After $12 \mathrm{~h}$, new serum-free medium was added for another $12 \mathrm{~h}$. Then, $10 \mu \mathrm{M}$ cytarabine was added for $48 \mathrm{~h}$. The culture medium was refreshed once every 2 days until the $10^{\text {th }}$ day of culture to harvest high purity rat neurons.

\section{MiR-124 transfection of rat RAs}

Purified RAs were seeded at a density of $5 \times 10^{5}$ cells/well in six-well plates for about $24 \mathrm{~h}$ before transfection. Thereafter, cells were transfected with different concentrations of ron-miR-124-3p mimic or corresponding negative control (RiboBio, China) using lipofectamine RNAiMAX (Invitrogen, 13778075). 
The cells were harvested at different time points after transfection for further detection of miRs, mRNAs, proteins, or for the following differentiation experiments.

\section{RNAi transfection of rat RAs}

Cell preparation before RNAi transfection was identical to that described for miR-124 transfection of RAs. Cells were transfected with 50 nM RNAi oligonucleotides (si-r-Sox9, si-r-Nfia, si-r-Hes1) or negative control siRNA (RiboBio, China) using lipofectamine RNAiMAX. After $24 \mathrm{~h}$, the medium was replaced by AM. Subsequently, cells were harvested at different time points for further experiments.

\section{Neuronal induction from rat cortical RAs}

After ron-miR-124-3p mimic transfection of RAs for $24 \mathrm{~h}$, the supernatant was switched to induced culture medium (DMEM/F12 medium supplemented with $2 \%$ FBS, $1 \%$ N2, $5 \mathrm{ng} / \mathrm{ml}$ bFGF, 1 uM ruxolitinib [Selleck, S1378], 10 uM SB203580 [Selleck, S1076], 10 uM forskolin [Selleck, S2449], 10 ng/ml BDNF [PeproTech, 450-02-10], $15 \mathrm{ng} / \mathrm{ml} \mathrm{GDNF}$ [PeproTech, 450-51-10], and 1\% penicillin-streptomycin). The culture medium was refreshed once every 2 days for 1 week for further experiments or switched to NM until the third week.

\section{Immunofluorescence staining}

After harvesting, all culture samples were fixed in 4\% paraformaldehyde for $20 \mathrm{~min}$, permeabilised with $0.2 \%$ Triton X-100 in phosphate-buffered saline (PBS) for $20 \mathrm{~min}$, and then incubated with $10 \%$ goat serum in PBS at room temperature for $1 \mathrm{~h}$. After blocking, samples were incubated with primary antibodies (Table S1) overnight at $4{ }^{\circ} \mathrm{C}$. After extensive washing, secondary antibodies (Table S1) in PBS were added for $1 \mathrm{~h}$ in the dark, followed by nuclear labelling with Hoechst33342 for $15 \mathrm{~min}$ at room temperature. Five randomly sampled images were captured for each culture using a laser scanning microscope (Olympus) and analysed using ImageJ software.

\section{Quantitative reverse transcription polymerase chain reaction (qRT-PCR) analysis}

Total miR and mRNA were extracted using a miR extraction kit (QIAGEN, 217004) and Trizol (Invitrogen, 15596026). Reverse transcription of $1000 \mathrm{ng}$ miRs from the total RNA was performed using the All-in-One miRNA first-strand cDNA Synthesis kit (GeneCopoeia, QP014); mRNA reverse transcription was performed using the TransScript-Uni One-Step gDNA Removal and cDNA Synthesis SuperMix (Transgen Biotech, AU311). MiR and mRNA PCR amplifications were performed on the 7300 Real Time PCR System using the All-in-One miRNA qPCR Detection kit (GeneCopoeia, QP011) and NovoStart SYBR qPCR SuperMix Plus kit (Novoprotein, E096-01B), respectively. Expression levels of rat U6 and GAPDH were used as internal controls for miRs and mRNAs; values were calculated as the reverse log of the $-\triangle \Delta C T$ from controls [28]. All assays were performed at least thrice. The primers used for the experiments (Table S2) were synthesised by Shanghai Sangon Biotech (Shanghai, China).

\section{Western blotting}


Total protein $(20 \mathrm{mg}$ ) was loaded onto $10 \%$ or $12 \%$ sodium dodecyl sulphate (SDS)-polyacrylamide gel electrophoresis (PAGE) gels and transferred to polyvinylidene fluoride membranes (Pall, BSP0161), which were blocked with $5 \%$ fat-free milk in Tris-buffered saline containing $0.1 \%$ Tween-20 (TBST) for $1 \mathrm{~h}$ at room temperature, followed by incubation with primary antibodies (Table S3) at $4{ }^{\circ} \mathrm{C}$ overnight. GAPDH was used as an internal control. After thorough washes with TBST, the membranes were incubated with peroxidase-conjugated secondary antibodies (Table S3) at room temperature for $1 \mathrm{~h}$. After washing, the signal was detected by chemiluminescence using the BeyoECL Plus (Beyotime, P0018) detection system and then analysed using ImageJ.

\section{RNA-sequencing (RNA-seq) library preparation and data analysis}

Samples were sent to Sangon Biotech Co., Ltd. (Shanghai, China) for library construction, Illumina sequencing, data pre-processing, and gene mapping. Total RNA extraction was performed using the Total RNA Extractor kit (Sangon Biotech, Shanghai). After RNA purity, concentration, and integrity assessment, $2 \mu \mathrm{g}$ high-quality RNA per sample were used as input material for RNA sample preparations. Sequencing libraries were generated using VAHTSTM mRNA-seq V2 Library Prep Kit for Illumina ${ }^{\circledR}$, and index codes were added to attribute sequences to each sample. Subsequently, FastQC (version 0.11.2) was used for evaluating the quality of the sequenced data. Raw reads were filtered using Trimmomatic (version 0.36) and the obtained sequence reads were trimmed and mapped to the reference genome using HISAT2 (version 2.1.0), followed by RNA-seq assessment. StringTie (version 1.3.3b) was used to calculate the transcripts per million (TPM) of protein-coding genes in each sample. For samples with three biological replicates, DESeq (version 1.12.4) was used to obtain the significant differentially expressed genes (sDEGs). Functional enrichment analysis was performed using the Gene Ontology (GO) and Kyoto Encyclopedia of Genes and Genomes (KEGG) enrichment analyses using TopGO (version 2.24.0) and ClusterProfiler (version 3.0.5), respectively.

\section{Co-immunoprecipitation}

Appropriate amount of immunoprecipitation lysis buffer (Beyotime, P0013) supplemented with protease inhibitor was added, and the resultant cell lysates were subject to immunoprecipitation with a specific antibody or IgG control (Beyotime, P7028) and protein A/G agarose beads (Beyotime, P2012) at $4{ }^{\circ} \mathrm{C}$ overnight, followed by thorough washes with lysis buffer. Subsequently, the bound proteins were eluted by heating the beads in $2 \%$ SDS gel-loading buffer at $95^{\circ} \mathrm{C}$ for $5 \mathrm{~min}$. Eluted proteins were resolved by SDS-PAGE and then detected by western blotting.

\section{Statistical analysis}

GraphPad Prism 7.0 was used for all statistical analyses, with the unpaired t-test for two-group comparisons and one-way or two-way analysis of variance for multiple-group comparisons. All experiments were repeated at least thrice. All data are represented as the mean \pm standard deviation (SD). For analyses, $p$-values $<0.05$ were considered statistically significant. 


\section{Results}

\section{Identification and characterisation of RAs from the postnatal rat cortex}

To avoid neural progenitor cell (NPC) contamination, only cerebral cortex regions where NPCs were not abundant were used. Cultured cells were expanded in medium containing TGF- $\beta 1$, which stimulated astrocytes to become RAs [29]. For about one week, until the cells reached $90 \%$ confluence, cytarabine was added to remove proliferating oligodendrocytes and microglia. After purification, most cells were typically star-shaped or polygonal, producing branching processes from the cell bodies (Figure 1A). More than $95 \%$ of the cells expressed the RA markers GFAP and S100 without DCX or MAP2 positive neuronal contamination (Figures 1B-1D). This suggested that the cultured cells were mainly RAs.

\section{Characterisation of RAs overexpressing miR-124}

To create RAs overexpressing miR-124, we transfected RAs with a miR-124 mimic (miR-124) or a negative control (NC-124). After a 48-h transfection, cell bodies in the miR-124 group became smaller and their branching processes slenderer compared with those in the NC-124 group (Figure 2A). qRT-PCR analysis demonstrated that miR-124 expression increased. The highest transfection efficiency was with $50 \mathrm{nM}$ miR-124 for $48 \mathrm{~h}$ (about 23.4 times higher than in the control) (Figures 2B and 2C). As expected, miR-124 overexpression reduced mRNA levels of the RA markers GFAP and S100 (Figure 2D). Additionally, GFAP and S100 protein expression was also downregulated after miR-124 overexpression (Figures 2E and 2F), which was consistent with the mRNA levels. These results suggested that we had successfully constructed miR-124 overexpressing RAs and that miR-124 overexpression suppressed the original astrocytic characteristics of RAs.

\section{RAs synergistically treated with miR-124, ruxolitinib, SB203580, and forskolin acquired neuronal properties}

Although miR-124 inhibited RA characteristics, it alone could not convert RAs into neurons. We then treated RAs overexpressing miR-124 with ruxolitinib, SB203580, and forskolin (Figure 3A). This synergistic combination dramatically converted RA morphology into neuron-like shapes after a 7-day treatment. The induced neuronal cell (iN) bodies became smaller and more compact and formed slender axon-like branching structures; no significant morphological changes were observed in the NC-124 control group (Figure 3B). Moreover, miR-124 group cells had smaller territory sizes and longer branching processes (Figures $3 \mathrm{C}$ and $3 \mathrm{D}$ ). Immunostaining demonstrated that early neuronal markers DCX and TUJ1 and mature neuronal markers MAP2 and NEUN were detectable in miR-124 group on the $7^{\text {th }}$ day of induction (Figures 3E-3G); the neurosynaptic junction marker SYN1 was detectable on the $21^{\text {st }}$ day (Figure $3 \mathrm{H}$ ). Based on neuronal marker expression and cell morphologies, the conversion efficiencies for DCX, TUJ1, MAP2, NEUN, and SYN1 were approximately $41.5 \%, 64.4 \%, 38.7 \%, 87.3 \%$, and $0.7 \%$, respectively (Figure $3 \mathrm{M})$. With regard to neuronal subtypes, our iNs were predominantly $\mathrm{CHAT}^{+}(25.9 \%)$ cholinergic and VGLUT1 ${ }^{+}(22.3 \%)$ glutamatergic, occasionally $\mathrm{TH}^{+}(6.1 \%)$ dopaminergic, but rarely 
$\operatorname{GAD} 67^{+}(1.4 \%)$ inhibitory neurons on the $7^{\text {th }}$ day of induction (Figures 3I-3L and $3 \mathrm{~N}$ ). These data suggested that rat cortical RAs acquired a neuronal fate after synergistic miR-124 and small molecules treatment, mainly forming cholinergic neurons.

\section{MiR-124, ruxolitinib, SB203580, and forskolin were indispensable for RA-to-neuron conversion}

To explore whether the same induction effects could be achieved using fewer components, different sets of miR-124 and small molecules were used for induction. The small molecules alone and miR-124 combined with at least one small molecule promoted the generation of DCX-positive neurons at day 7 , but conversion efficiency was the highest when miR-124 and the three small molecules were added synergistically (Figures 4A and 4B). MAP2 immunostaining results were also consistent (Figures 4C and 4D). As JAK/STAT3, P38 MAPK, and ERK1/2 signalling is involved in RA migration, astrogliosis, and proliferation [30-32], increased phosphorylation levels would enhance astrocyte activation. Therefore, we assessed the phosphorylation levels in the iNs. Western blot results at day 7 of induction revealed that phosphorylation levels were most downregulated when miR-124 and the three small molecules were added synergistically (Figures $4 \mathrm{E}$ and $4 \mathrm{~F}$ ); this also reduced RA reactivity and benefited neuronal transdifferentiation the most. These results suggested that the synergistic effects of miR-124 and the three small molecules best promoted RA-to-neuron conversion.

\section{Gene expression profile of iNs was closer to that of cortical neurons than of RAs}

To examine the differences between RAs, iNs, and rat neurons at the genetic level, we first isolated rat cortical neurons and maintained them in a defined, serum-free NM. At day 10 after purification, the cells exhibited a typical neuronal morphology (Figure 5A). Most cells were immune-positive for TUJ1 and MAP2 (Figure 5B), suggesting that the isolated cells were mainly neurons. As miR-124 expression gradually increases during neuronal differentiation and maturation [33], we assessed miR-124 expression in iNs. At day 7 of induction, total miR-124 expression was 23.9 times higher than that in control group (Figure 5C). Considering that exogenous miR-124 decreases gradually (Figure 2C), the total miR-124 overexpression was mainly caused by increased endogenous miR-124, further indicating RA-to-neuron conversion. To identify the gene expression profiles of RAs, iNs (day 7), and rat neurons (day 10), RNAseq analyses were performed. The number of sDEGs (TPM $\geq 5$ in at least one sample, |FoldChange|>2, qvalue $<0.05$ ) between iNs and RAs (3065) was more than that between iNs and rat neurons (2695) (Figure 5D), indicating fewer differences between the latter two. Consistently, hierarchical cluster analysis indicated that the gene expression profile of iNs was more similar to that of rat neurons than of RAs (Figure 5E), similar to the qRT-PCR results (Figure 5F). These results suggested that RAs silenced their astrocytic transcriptional programs and adopted a neuronal identity during induction.

As the induction efficiency was incomplete, we were interested in whether prolonged induction time or environmental factors would affect the fate of the remaining RAs. Therefore, we cultured the 21-day iNs in AM and NM. On the third day of continuous culturing, no morphological changes were observed. On the $11^{\text {th }}$ day, cell morphology in NM was still unchanged, similar to that of rat neurons. However, cell 
numbers in AM increased, and the morphology was similar to that of RAs (Additional file 1: Figure S1A), which might be because serum exposure allowed the remaining RAs to proliferate. These results indicated that prolonged exposure and a neuronal environment could not change the fate of non-neuronal transformed RAs. Subsequently, we assessed the gene expression of these cells, RAs, and rat neurons. The genetic pattern of cells in AM resembled that of RAs and that of cells in NM resembled that of rat neurons (Additional file 1: Figure S1B).

\section{Transcriptome analysis of iNs and RAs}

To explore the molecular and cellular changes of iNs compared with their initial cells, we further analysed the transcriptome of iNs and RAs. At day 7 of induction, the transcriptome scatterplot analysis revealed that, of the 3065 sDEGs, 1820 were upregulated and 1245 were downregulated (Figure 6A). The expression of RA-enriched genes (e.g., Gfap, Aldh1/1, Vim, Nes, Slit2, Axin2, and Mmp2) was decreased, while that of neuron-enriched genes (e.g., Dcx, Tuj1, Syt1, Kcnc1, Map2, Syn1, and Ascl1) was significantly increased. Additionally, expression of genes involved in brain development or cholinergic neuron differentiation (e.g., Foxg 1, Bc/11b, and Myt11) was also significantly upregulated. Further, qRT-PCR results confirmed expression patterns of the selected genes, which were consistent with the RNA-seq results (Figure 6B). The GO analysis (Biological process, Cellular component, and Molecular function) showed that the upregulated genes were significantly enriched in neuron projection morphogenesis, neuron differentiation, axonogenesis, and synaptic vesicle (Figure 6C), and the downregulated genes were significantly enriched in cell proliferation, cell migration, cell-substrate adhesion, and proteoglycan binding (Figure 6D), suggesting that the astrogliosis and scar formation properties of RAs had weakened, while neuronal characteristics had increased. Additionally, KEGG analysis revealed that upregulated cyclic guanosine monophosphate-dependent protein kinase $\mathrm{G}$ and cyclic adenosine monophosphate (cAMP) signalling, soluble NSF attachment protein receptor interactions in vesicular transport, and other upregulated signals were mainly involved in neuron development, axon formation, and neurotransmitter release (Figure 6E), while downregulated MAPK signalling, cell adhesion molecules, PI3K-AKT signalling, and other downregulated signals were related to astrogliosis and glial scarring of RAs (Figure 6F), further pointing to RA-to-neuron conversion. Together, the transcriptional regulatory network of RAs was reprogrammed toward that of neuronal lineage under our induction.

\section{MiR-124 regulated neuronal differentiation of RAs via the SOX9-NFIA-HES1 axis}

To clarify the molecular mechanism involved in the regulatory role of miR-124, we focused on the NOTCH downstream effectors SOX9 and NFIA, characteristic RA TFs [34], as well as HES1, whose oscillations are related to neurogenesis [35]. MiR-124 overexpression notably repressed SOX9, NFIA, and HES1 expression (Figures 7A and 7B), indicating the regulatory effect of miR-124 on them. Considering that Sox9 is a target of miR-124 [36], we explored the relationship between SOX9, NFIA, and HES1 in RAs. After transfection with si-Sox9 (Additional file 1: Figure S2A), Sox9 mRNA and protein levels were significantly reduced (Figures 7C-7E). Moreover, NFIA and HES1 expression was also downregulated with reductions in SOX9 levels (Figures 7D and 7E), suggesting that SOX9 regulated NFIA and HES1. Additionally, a physical 
association between SOX9 and NFIA confirmed by co-immunoprecipitation further indicated that SOX9 directly downregulated NFIA (Figure 7F). Subsequently, we transfected RAs with si-Nfia (Additional file 1: Figure S2B), and Nfia mRNA level and protein expression were remarkably decreased (Figures 7G-7I). Moreover, HES1 also decreased upon reduced levels of NFIA (Figures 7H and 7I), which reduces HES1 expression by binding to the Hes1 promoter region [37], suggesting the direct repression of NFIA on HES1. Altogether, miR-124 could downregulate HES1 expression by targeting the SOX9-NFIA-HES1 axis in RAs. To explore whether this axis was related to neurodifferentiation, we transfected RAs with si-Hes1 (Additional file 1: Figure S2C), which significantly downregulated Hes 1 mRNA expression (Figures 7J-7L). Furthermore, the mRNA expression of $A s c / 1$, a gene related to neuronal differentiation, was significantly elevated by Hes 1 silencing (Figure 7J), revealing the negative regulation of Hes 1 in neuronal differentiation. Simultaneously, we detected the effect of Sox9 or Nfia inhibition on Asc/1. The results showed that Sox9 or Nfia inhibition could also increase Ascl1 mRNA expression (Additional file 1: Figure S2D), but not as much as Hes1 inhibition, further indicating the indispensable role of Hes 1 in this regulatory axis. These results suggested that in RAs, miR-124 inhibited NOTCH signalling by targeting the SOX9-NFIA-HES1 axis, thereby promoting RA-to-neuron conversion.

\section{HES1 was involved in the RA-to-neuron conversion co-regulated by miR-124, ruxolitinib, SB203580, and forskolin}

To decipher whether the SOX9-NFIA-HES1 axis operated during the induction after the small molecules were added, we first induced the aforementioned si-Hes1 RAs with the three small molecules for 3 days, and compared their HES1 expression with that in RAs, RAs treated with the three small molecules only, and RAs treated with both miR-124 and the three small molecules. Western blotting showed that HES1 expression was significantly suppressed in the miR-124 and small molecules group (Figures 8A and 8B). Immunostaining for DCX demonstrated that conversion efficiency in the miR-124 and small molecules group was better than those in other groups (Figures $8 \mathrm{E}$ [a-d] and 8F). Given that the miR-124 and small molecules group had the lowest HES1 expression but the best conversion efficiency; we speculated whether HES1 expression determined differences in conversion efficiency. We then applied DAPT, a NOTCH pathway inhibitor, to further suppress HES1 expression. For better effects, we also prolonged induction time to 7 days. As expected, DAPT application further suppressed HES1 expression in the miR124 and small molecules group (Figures $8 \mathrm{C}$ and 8D), but conversion efficiency was still lower than that in the miR-124 and small molecules group without added DAPT (Figures 8E [e-h] and 8G), demonstrating that apart from suppressing HES1 expression, the combination might also regulate neuronal conversion by other means. These results suggested that HES1 expression was involved in RA-to-neuron conversion co-regulated by the miR-124 and small molecule cocktail, but this was not the only mechanism.

\section{Discussion}


Astrocytes, a major cell population in the mammalian CNS, play multiple important roles in CNS pathophysiology $[38,39]$. When pathological conditions affect the CNS, astrocytes become reactive and are characterised by morphological changes (hypertrophy and process extension) and the upregulation of intermediate filament proteins such as GFAP and VIMENTIN [3, 40]. Moreover, the production of chondroitin sulphate proteoglycans and release of pro-inflammatory cytokines further drives RAs to form glial scars, inhibiting neuronal regeneration [41].

Recently, the use of diverse small molecule cocktails to reprogram astrocytes into neuronal cells has achieved considerable success $[13,14]$. Yin et al. proved that using three to four small molecules (SB431542, CHIR99021, LDN193189, and DAPT) or their respective functional analogues to modulate the TGF- $\beta$ /SMAD, BMP, NOTCH, and WNT pathways was sufficient to reprogram human foetal astrocytes into neurons [14]. However, RA characteristics are also regulated by other signals, such as the JAK/STAT and P38 MAPK pathways and post-transcriptional levels of several non-coding RNAs. Here, we developed a new miR-124, ruxolitinib, SB203580, and forskolin cocktail, proving that this combination could significantly alter RA characteristics and promote RA-to-neuron conversion.

Studies have revealed the functional significance of miRs in regulating CNS development [42]. MiR-124, one of the most highly expressed miRs in the mammalian brain, is closely associated with the regulation of neurogenesis and neuronal differentiation [43]. Given that both astrocytes and neurons are derived from NSCs and that miR-124 is a neuronal fate determinant during neurodevelopment [44], we hypothesised that differential miR-124 expression might affect their distinct cell fates. Indeed, we found that miR-124 expression in astrocytes, especially RAs, was much lower than that in neurons (Additional file 1: Figure S3). Moreover, GFAP, S100, SOX9, and NFIA expression was significantly downregulated after miR-124 overexpression, indicating that miR-124 upregulation could efficiently suppress inherent RA characteristics and might promote RA-to-neuron conversion. Additionally, as each miR regulates hundreds of target genes [45], and Smad4 (involved in both the TGF- $\beta$ and BMP pathways), Dact1, and Axin1 (negative regulators of the WNT/ $\beta$-catenin cascade) are all targets of miR-124 [46-49], we hypothesised that miR-124 might replace TGF- $\beta$ and BMP pathway inhibitors as well as WNT/ $\beta$-catenin pathway activators in RAs and implement functions similar to those of the SB431542, LDN193189, and CHIR99021 combination during RA-to-neuron reprogramming. As expected, when we randomly selected SB431542 to add to the miR-124 and three small-molecule cocktail, no significant increase in DCXpositive conversion efficiency was observed (Additional file 1: Figures S4A and S4B). Considering its numerous targets, miR-124 might promote RA-to-neuron conversion through other unknown mechanisms, and eventually the effects of our cocktail were better than of the above three chemicals. However, miR124 itself was not sufficient to reprogram RAs into neurons in vitro, suggesting that signals other than TGF- $\beta$, BMP, and WNT, such as JAK/STAT [3] and P38 MAPK [31], that are crucial for maintaining RA characteristics, might be constantly activated and impair the transformation process. We thus hypothesised that additional inhibition of these signals might achieve more significant conversion efficiency. 
JAK/STAT pathway activation is reported to be involved in CNS injuries, especially gliogenesis and glial scarring formation [30,50]. Moreover, Jak3 silencing or Stat3 ablation promoted NPC neuronal differentiation and NSC neurogenesis, respectively [51, 52], revealing the neuronal promotion of JAK/STAT inhibition. Therefore, we speculated that JAK/STAT pathway suppression offers great possibilities for inhibiting inherent RA properties and promoting RA-to-neuron conversion. As expected, the conversion efficiency was significantly increased after treatment with ruxolitinib and miR-124. To the best of our knowledge, this is the first time a JAK/STAT pathway inhibitor has been used for neuronal induction.

P38 MAPK and ERK1/2 activation is also associated with astrogliosis [53]. During reactive gliosis, ASK1 phosphorylation is enhanced, and P38 MAPK relays the signal to astrocytes for activation and glial scar formation both in vitro and vivo $[54,55]$. Thus, suppressing P38 MAPK signalling was more likely to attenuate RA characteristics and favour neuronal induction. Here we utilised SB203580, a common P38 inhibitor, to repress RA characteristics and promote neuronal conversion. Furthermore, forskolin is a wellknown CAMP and PRKACA activator and promotes neuron survival and neuronal conversion efficiency [56-58]. As expected, the three small molecules promoted RA-to-neuron conversion, albeit inefficiently. However, when miR-124 was also added, efficient conversion was obtained, further indicating the importance of combinatory functions.

Although the functional mechanisms of the three small molecules are clear, the function of miR-124 is more complicated. Here, we mainly focus on miR-124 and NOTCH signalling, a highly conserved morphogenic pathway that regulates NSC proliferation, self-renewal, and neuronal differentiation [59]. Attenuated $\mathrm{NOTCH} 1$ signalling after stroke is necessary for striatal astrocyte neurogenesis in mice [60], indicating that RA-to-neuron conversion is related to NOTCH signalling. Here, we revealed that miR-124 promotes neuronal differentiation of RAs by targeting the SOX9-NFIA-HES1 axis and eventually elevates the expression of Ascl1 (Fig. 9), a proneural target gene of Hes 1 that promotes neural differentiation [61]. This was consistent with previous studies showing that expression of the neural TF ASCL1 alone could reprogram astrocytes into neurons [62]. Nevertheless, persistent miR-124 overexpression alone did not produce DCX- or MAP2-positive RA-derived neurons. This might be because neurodifferentiation is promoted only when proneural activity is high enough [63]. Although Asc/1 was upregulated in RAs, the levels were not high enough for neuronal conversion. Moreover, HES1 expression disappears in differentiating neurons, leading to sustained ASCL1 expression, inducing neuronal differentiation [64]. In our study, miR-124 indirectly inhibited HES1 but failed to eliminate its expression. Thereafter, when the small molecules were added to regulate additional RA signals, we obtained DCX- and MAP2-positive iNs; however, when DAPT was added to further suppress HES1 expression in RAs, no more DCX-positive iNs were obtained. This might be because HES1 is a repressor-type basic helix-loop-helix protein, while ASCL1 is of the activator-type. When NOTCH signalling is inhibited, decreased HES1 expression upregulates ASCL1 to simultaneously promote neuronal fate determination and NOTCH ligands expression, activating NOTCH signalling and upregulating HES1 expression in neighbouring cells to maintain them in an undifferentiated state [65]. Therefore, HES1 and ASCL1 regulate each other, only allowing some cells to undergo neuronal differentiation. This may also explain why the remaining undifferentiated RAs in our 
study could not acquire a neuronal identity even with prolonged induction. Of course, the synergy between miR-124 and the other three small molecules is extremely complex, and this analysis is from the limited perspective of HES1 expression alone. Whether this synergism regarding RA-to-neuron conversion could function in other ways needs to be further explored.

The neuronal subtypes after conversion from astrocytes depend on the combinations of inducing chemicals. Previous nine- and four-molecule cocktails mainly converted astrocytes into glutamatergic neurons through upregulation of NEUROD1 and NGN2 $[14,66]$. Here, our RNA-seq results showed that Foxg1 and Myt1/mRNA expression was significantly upregulated. FOXG1 is one of the earliest TFs specifically induced in forebrain NPCs [67]. MYT1L, the only known TF specifically expressed in all neurons throughout life, is critical for maintaining neuronal identity $[68,69]$. Thus, our induction modulated RAs to obtain a neuronal identity via upregulating Foxg1 and Myt1l. Interestingly, unlike with other protocols, many cholinergic neurons appeared in our experiments. The qRT-PCR results also revealed that expression of genes involved in cholinergic neuron differentiation (e.g., Chat, Lhx8, Lhx6, and Tbr1) was significantly upregulated in these iNs (Additional file 1: Figure S5). The cholinergic hypothesis of Alzheimer's disease (AD) proposes that brain acetylcholine depletion is a primary cause of cognitive decline in advanced age and $A D[70,71]$. Moreover, cholinergic nuclei are differently affected by the pathogenetic mechanisms underlying major neurodegenerative diseases such as AD, PD, and Huntington disease [70]. Given that astrocyte reactivity is a hallmark of the pathological CNS, RA-derived cholinergic neurons might be potential targets for the treatment of neurodegenerative diseases. Recently, Liang et al. reported that MYT1L could directly reprogram human brain vascular pericytes into cholinergic neurons [72], indicating the Myt1/ expression in our experiments might be essential for converting and maintaining the cholinergic neuron phenotype. Although these mechanisms remained to be further explored, we believe that the different effects depended on our unique induction cocktail.

Glial scars formed by RAs after CNS injuries ultimately impair neuronal regeneration; therefore, ameliorating the cellular properties of glial scars is essential for neural repair. Although $41.5 \%$ RAs in this study were successfully converted into DCX-positive neurons, with continuous miR-124 and small molecules treatment, the remaining undifferentiated RAs would lose their reactivity to form glial scars, and further contact the iNs to support their maturation. Collectively, utilising our cocktail simultaneously achieved the two goals of reprogramming RAs into neurons and repressing RA cellular characteristics, which is significant for the treatment of CNS injuries.

Although the use of small molecules to reprogram cells has achieved remarkable success, they should be non-toxic and approved after clinical safety trials. Ruxolitinib, the first clinically effective, selective JAK1/2 inhibitor approved by the Food and Drug Administration, can penetrate the blood-brain-barrier and reduce astrogliosis in mice with HIV encephalitis when systemically administered [73]. Additionally, losmapimod, a P38 MAPK $\alpha$ and $\beta$ isoform inhibitor functionally analogous to SB203580, has entered stage I clinical trials for treating facioscapulohumeral muscular dystrophy. Forskolin is a natural compound used for centuries in traditional medicine. These chemicals selected for our formula or their functional analogues are closer to clinical application and more feasible for use. 


\section{Conclusions}

In summary, this study shows that the combination of miR-124, ruxolitinib, SB203580, and forskolin can successfully reprogram rat RAs into mainly cholinergic neurons in vitro. Whether or not this combination can effectively reprogram human RAs into neurons and have similar conversion efficiency in vivo requires further investigation. Altogether, our novel RA-to-neuron reprogramming approach provides new clues for inhibiting glial scars and repairing CNS damage.

\section{Declarations}

\section{Ethics approval:}

The animal study has been reviewed and approved by the Ethics Committee of Jilin University (Ethical approval code: 2015-19).

Consent for publication:

Not applicable.

\section{Availability of data and materials:}

The datasets used and/or analysed during the current study are available from the corresponding author (Guangfan Chi, guangfan130@jlu.edu.cn) on reasonable request.

\section{Competing interests:}

The authors declare that they have no competing interests.

\section{Funding:}

This work was supported by the National Natural Science Foundation of China [Grant number 81571199, Grant number 81870974] and the Fundamental Research Funds for the Central Universities, JLU. The funding body had no role in the design of the study and collection, analysis, and interpretation of data and in writing the manuscript.

\section{Authors' contributions:}

$\mathrm{GC}, \mathrm{CQ}$, and $\mathrm{YL}$ conceived the study, designed the experiments, and together with $\mathrm{YZ}$ revised the manuscript. YZ performed the cellular and molecular experiments in vitro, with help from ZH and JX. KH, YY, SL, and LC provided reagents and conducted the data analysis and interpretation. YZ wrote the manuscript. All authors approved the final version of the manuscript. 


\section{Acknowledgements:}

We thank Shanghai Sangon Biotech Co., Ltd., for RNA-seq analysis and Guangzhou RiboBio Co., Ltd., for designing the miR mimics and si-RNA. We also thank Editage for English language editing.

\section{Abbreviations}

CNS, central nervous system; RA, reactive astrocyte; NSC, neural stem cell; TF, transcription factor; DAPT, 2, 4-diamino-5-phenylthiazole; RNA, ribonucleic acid; miR, microRNA; mRNA, messenger ribonucleic acid; PD, Parkinson's disease; NPC, neural progenitor cell; iN, induced neuronal cell; FBS, foetal bovine serum; PBS, phosphate-buffered saline; TBST, Tris-buffered saline containing $0.1 \%$ Tween-20; SDS, sodium dodecyl sulphate; PAGE, polyacrylamide gel electrophoresis; qRT-PCR, quantitative reverse transcription polymerase chain reaction; RNA-seq, RNA-sequencing; TPM, transcripts per million; sDEG, significant differentially expressed gene; GO, Gene Ontology; KEGG, Kyoto Encyclopedia of Genes and Genomes; SD, standard deviation; NM, neuron conditioned medium; AM, RA conditioned medium; cAMP, cyclic adenosine monophosphate; $A D$, Alzheimer's disease

\section{References}

1. Ouyang W, et al. Moderate injury in motor-sensory cortex causes behavioral deficits accompanied by electrophysiological changes in mice adulthood. PLoS One. 2017;12(2):e0171976.

2. Bhowmick S, et al., Neurodegeneration and Sensorimotor Deficits in the Mouse Model of Traumatic Brain Injury. Brain Sci, 2018. 8(1).

3. Okada S, et al. Astrocyte reactivity and astrogliosis after spinal cord injury. Neurosci Res. 2018;126:39-43.

4. Wang $\mathrm{H}$, et al. Portrait of glial scar in neurological diseases. Int J Immunopathol Pharmacol. 2018;31:2058738418801406.

5. McMurran CE, et al. Clinical implications of myelin regeneration in the central nervous system. Expert Rev Neurother. 2018;18(2):111-23.

6. Tsunemoto R, et al. Diverse reprogramming codes for neuronal identity. Nature. 2018;557(7705):375-80.

7. Kanakov 0 , et al. Astrocyte-induced positive integrated information in neuron-astrocyte ensembles. Phys Rev E. 2019;99(1-1):012418.

8. Farhy-Tselnicker I, Allen NJ. Astrocytes, neurons, synapses: a tripartite view on cortical circuit development. Neural Dev. 2018;13(1):7.

9. Buganim Y, Faddah DA, Jaenisch R. Mechanisms and models of somatic cell reprogramming. Nat Rev Genet. 2013;14(6):427-39.

10. Heinrich C, Gotz M, Berninger B. Reprogramming of postnatal astroglia of the mouse neocortex into functional, synapse-forming neurons. Methods Mol Biol. 2012;814:485-98. 
11. Su Z, et al. In vivo conversion of astrocytes to neurons in the injured adult spinal cord. Nat Commun. 2014;5:3338.

12. Lee $C$, Robinson M, Willerth SM. Direct Reprogramming of Glioblastoma Cells into Neurons Using Small Molecules. ACS Chem Neurosci. 2018;9(12):3175-85.

13. Gao L, et al. Direct Generation of Human Neuronal Cells from Adult Astrocytes by Small Molecules. Stem Cell Reports. 2017;8(3):538-47.

14. Yin JC, et al. Chemical Conversion of Human Fetal Astrocytes into Neurons through Modulation of Multiple Signaling Pathways. Stem Cell Reports. 2019;12(3):488-501.

15. Lagos-Quintana $M$, et al. Identification of novel genes coding for small expressed RNAs. Science. 2001;294(5543):853-8.

16. Abernathy DG, et al. MicroRNAs Induce a Permissive Chromatin Environment that Enables Neuronal Subtype-Specific Reprogramming of Adult Human Fibroblasts. Cell Stem Cell. 2017;21(3):332-48.e9.

17. Ghasemi-Kasman $M$, et al. miR-302/367-induced neurons reduce behavioral impairment in an experimental model of Alzheimer's disease. Mol Cell Neurosci. 2018;86:50-7.

18. Mishima T, et al. RT-PCR-based analysis of microRNA (miR-1 and - 124) expression in mouse CNS. Brain Res. 2007;1131(1):37-43.

19. Sun $Y$, et al. An updated role of microRNA-124 in central nervous system disorders: a review. Front Cell Neurosci. 2015;9:193.

20. Jiao S, et al. miR-124 promotes proliferation and neural differentiation of neural stem cells through targeting DACT1 and activating Wnt/beta-catenin pathways. Mol Cell Biochem. 2018;449(1-2):30514.

21. Xue $Q$, et al. miR-9 and miR-124 synergistically affect regulation of dendritic branching via the AKT/GSK3beta pathway by targeting Rap2a. Sci Rep. 2016;6:26781.

22. Yang J, et al. Exosome Mediated Delivery of miR-124 Promotes Neurogenesis after Ischemia. Mol Ther Nucleic Acids. 2017;7:278-87.

23. Yoo AS, et al. MicroRNA-mediated conversion of human fibroblasts to neurons. Nature. 2011;476(7359):228-31.

24. Lim LP, et al. Microarray analysis shows that some microRNAs downregulate large numbers of target mRNAs. Nature. 2005;433(7027):769-73.

25. Zhou H, et al. Glia-to-Neuron Conversion by CRISPR-CasRx Alleviates Symptoms of Neurological Disease in Mice. Cell. 2020;181(3):590-603.e16.

26. Qian $\mathrm{H}$, et al. Reversing a model of Parkinson's disease with in situ converted nigral neurons. Nature. 2020;582(7813):550-6.

27. Mokabber $\mathrm{H}$, Najafzadeh $\mathrm{N}$, Mohammadzadeh Vardin $\mathrm{M}$, miR-124 promotes neural differentiation in mouse bulge stem cells by repressing Ptbp 1 and Sox9. J Cell Physiol, 2019. 234(6): p. 8941-8950.

28. Pfaffl MW. A new mathematical model for relative quantification in real-time RT-PCR. Nucleic Acids Res. 2001;29(9):e45. 
29. Lee HS, et al. Meteorin is upregulated in reactive astrocytes and functions as a negative feedback effector in reactive gliosis. Mol Med Rep. 2015;12(2):1817-23.

30. Ben Haim L, et al. The JAK/STAT3 pathway is a common inducer of astrocyte reactivity in Alzheimer's and Huntington's diseases. J Neurosci. 2015;35(6):2817-29.

31. Roy Choudhury G, et al. Involvement of p38 MAPK in reactive astrogliosis induced by ischemic stroke. Brain Res. 2014;1551:45-58.

32. Jia $\mathrm{M}$, et al. Insulin and heparin-binding epidermal growth factor-like growth factor synergistically promote astrocyte survival and proliferation in serum-free medium. J Neurosci Methods. 2018;307:240-7.

33. Smirnova L, et al. Regulation of miRNA expression during neural cell specification. Eur J Neurosci. 2005;21(6):1469-77.

34. Kang P, et al. Sox 9 and NFIA coordinate a transcriptional regulatory cascade during the initiation of gliogenesis. Neuron. 2012;74(1):79-94.

35. Ochi S, et al., Oscillatory expression of Hes 1 regulates cell proliferation and neuronal differentiation in the embryonic brain. Development, 2020. 147(4).

36. Zhou F, et al., Screening the expression characteristics of several miRNAs in G93A-SOD1 transgenic mouse: altered expression of miRNA-124 is associated with astrocyte differentiation by targeting Sox2 and Sox9. J Neurochem, 2018. 145(1): p. 51-67.

37. Piper M, et al. NFIA controls telencephalic progenitor cell differentiation through repression of the Notch effector Hes1. J Neurosci. 2010;30(27):9127-39.

38. Boddum K, et al. Astrocytic GABA transporter activity modulates excitatory neurotransmission. Nat Commun. 2016;7:13572.

39. Bittern J, et al., Neuron-glia interaction in the Drosophila nervous system. Dev Neurobiol, 2020.

40. Burda JE, Sofroniew MV. Reactive gliosis and the multicellular response to CNS damage and disease. Neuron. 2014;81(2):229-48.

41. Moeendarbary E, et al. The soft mechanical signature of glial scars in the central nervous system. Nat Commun. 2017;8:14787.

42. Cao DD, Li L, Chan WY. MicroRNAs: Key Regulators in the Central Nervous System and Their Implication in Neurological Diseases. Int J Mol Sci, 2016. 17(6).

43. Kutsche LK, et al. Combined Experimental and System-Level Analyses Reveal the Complex Regulatory Network of miR-124 during Human Neurogenesis. Cell Syst. 2018;7(4):438-52.e8.

44. Akerblom M, et al. MicroRNA-124 is a subventricular zone neuronal fate determinant. J Neurosci. 2012;32(26):8879-89.

45. Guarnieri DJ, DiLeone RJ. MicroRNAs: a new class of gene regulators. Ann Med. 2008;40(3):197208.

46. Zhang Z, et al. MicroRNA-124 inhibits the proliferation of C6 glioma cells by targeting Smad4. Int J Mol Med. 2017;40(4):1226-34. 
47. Zu L, et al. The feedback loop between miR-124 and TGF-beta pathway plays a significant role in non-small cell lung cancer metastasis. Carcinogenesis. 2016;37(3):333-43.

48. Jiao S, et al. miR-124 promotes proliferation and neural differentiation of neural stem cells through targeting DACT1 and activating Wnt/ $\beta$-catenin pathways. Mol Cell Biochem. 2018;449(1-2):305-14.

49. Lu Y, et al. MiR-124 regulates transforming growth factor-beta1 induced differentiation of lung resident mesenchymal stem cells to myofibroblast by repressing Wnt/beta-catenin signaling. Dev Biol. 2019;449(2):115-21.

50. Tapia VS, Herrera-Rojas M, Larrain J. JAK-STAT pathway activation in response to spinal cord injury in regenerative and non-regenerative stages of Xenopus laevis. Regeneration (Oxf). 2017;4(1):21-35.

51. Kim YH, et al. Differential regulation of proliferation and differentiation in neural precursor cells by the Jak pathway. Stem Cells. 2010;28(10):1816-28.

52. Cao F, et al. Conditional deletion of Stat3 promotes neurogenesis and inhibits astrogliogenesis in neural stem cells. Biochem Biophys Res Commun. 2010;394(3):843-7.

53. Wang $\mathrm{CY}$, Yang SH, Tzeng SF. MicroRNA-145 as one negative regulator of astrogliosis. Glia. 2015;63(2):194-205.

54. Cheon SY, et al. Knockdown of apoptosis signal-regulating kinase 1 affects ischaemia-induced astrocyte activation and glial scar formation. Eur J Neurosci. 2016;43(7):912-22.

55. Li T, et al. ASK1 phosphorylation regulates astrocytic reactive gliosis in vitro and in vivo. Neurosci Lett. 2020;716:134675.

56. Smith DK, et al. Small Molecules Modulate Chromatin Accessibility to Promote NEUROG2-Mediated Fibroblast-to-Neuron Reprogramming. Stem Cell Reports. 2016;7(5):955-69.

57. Gascon S, et al. Identification and Successful Negotiation of a Metabolic Checkpoint in Direct Neuronal Reprogramming. Cell Stem Cell. 2016;18(3):396-409.

58. Liu ML, et al. Small molecules enable neurogenin 2 to efficiently convert human fibroblasts into cholinergic neurons. Nat Commun. 2013;4:2183.

59. Roese-Koerner B, Stappert L, Brustle O. Notch/Hes signaling and miR-9 engage in complex feedback interactions controlling neural progenitor cell proliferation and differentiation. Neurogenesis (Austin). 2017;4(1):e1313647.

60. Magnusson JP, et al. A latent neurogenic program in astrocytes regulated by Notch signaling in the mouse. Science. 2014;346(6206):237-41.

61. Sueda R, et al. High Hes1 expression and resultant Ascl1 suppression regulate quiescent vs. active neural stem cells in the adult mouse brain. Genes Dev. 2019;33(9-10):511-23.

62. Liu Y, et al. Ascl1 Converts Dorsal Midbrain Astrocytes into Functional Neurons In Vivo. J Neurosci. 2015;35(25):9336-55.

63. Boareto M, Iber D, Taylor V. Differential interactions between Notch and ID factors control neurogenesis by modulating Hes factor autoregulation. Development. 2017;144(19):3465-74. 
64. Imayoshi I, Kageyama R. bHLH factors in self-renewal, multipotency, and fate choice of neural progenitor cells. Neuron. 2014;82(1):9-23.

65. Kageyama R, et al. Roles of bHLH genes in neural stem cell differentiation. Exp Cell Res. 2005;306(2):343-8.

66. Zhang L, et al. Small Molecules Efficiently Reprogram Human Astroglial Cells into Functional Neurons. Cell Stem Cell. 2015;17(6):735-47.

67. Cargnin F, et al. FOXG1 Orchestrates Neocortical Organization and Cortico-Cortical Connections. Neuron. 2018;100(5):1083-96.e5.

68. Mall M, et al. Myt1l safeguards neuronal identity by actively repressing many non-neuronal fates. Nature. 2017;544(7649):245-9.

69. Matsushita F, et al. Spatiotemporal expression pattern of Myt/NZF family zinc finger transcription factors during mouse nervous system development. Dev Dyn. 2014;243(4):588-600.

70. Pepeu G, Grazia M, Giovannini. The fate of the brain cholinergic neurons in neurodegenerative diseases. Brain Res. 2017;1670:173-84.

71. Douchamps V, Mathis C, A second wind for the cholinergic system in Alzheimer's therapy. Behav Pharmacol, 2017. 28(2 and 3-Spec Issue): p. 112-123.

72. Liang XG, et al. Myt1l induced direct reprogramming of pericytes into cholinergic neurons. CNS Neurosci Ther. 2018;24(9):801-9.

73. Haile WB, et al. The Janus kinase inhibitor ruxolitinib reduces HIV replication in human macrophages and ameliorates HIV encephalitis in a murine model. Neurobiol Dis. 2016;92(Pt B):137-43.

\section{Figures}


A
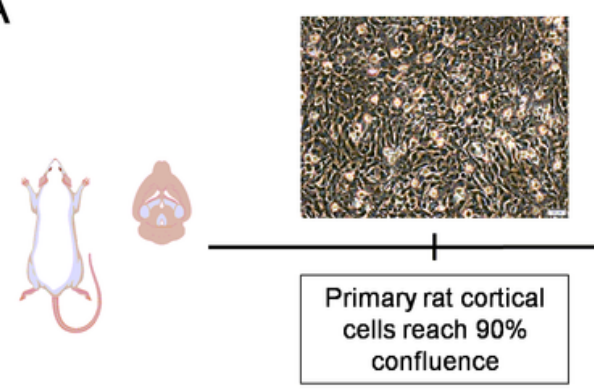

B

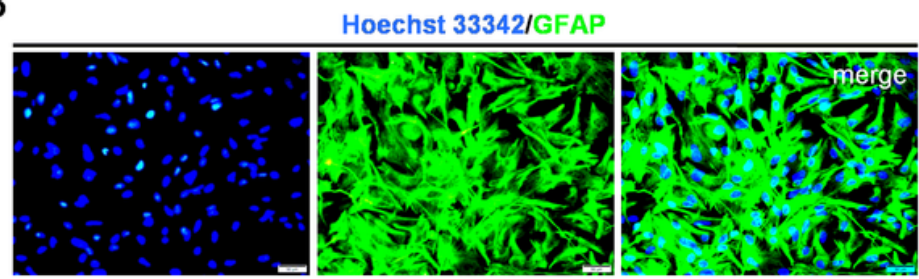

Hoechst 33342/S100

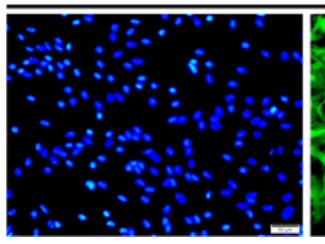

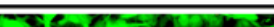

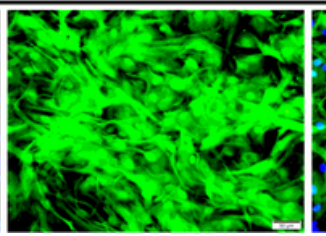

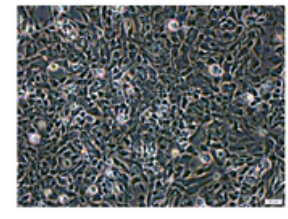

Shaking the cells at $37^{\circ} \mathrm{C}(280 \mathrm{rpm} / \mathrm{min}$,

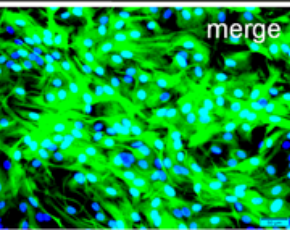
$10 \mathrm{~h} /$ day) for 2 days

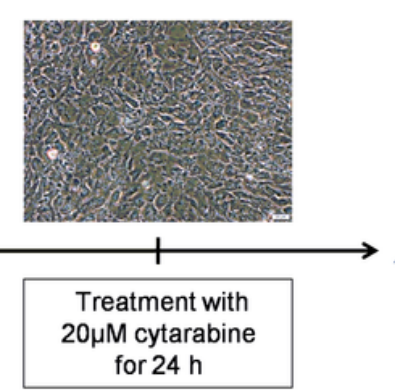

C

C

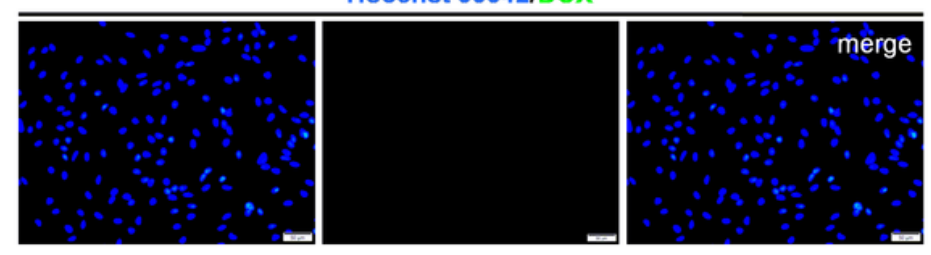

Hoechst 33342/MAP2

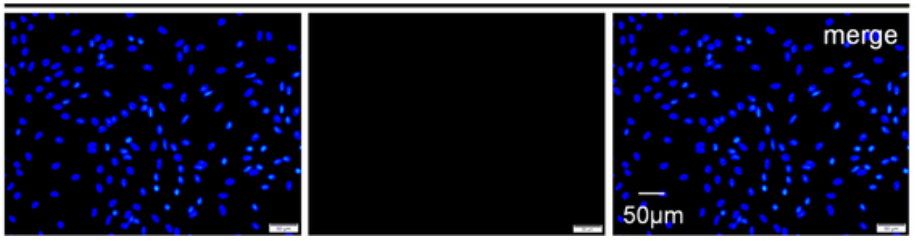

Figure 1

Morphological and biochemical characteristics of primary rat cortical RAs (A) Purification processes of primary RAs and corresponding cell morphologies at different time points in vitro. (B-C) Immunostaining shows that the majority of cultured cells expressed the RA markers, GFAP and S100, but not the neuronal markers DCX and MAP2. (D) Statistical analysis shows that $>95 \%$ of all cells were GFAP- and S100positive RAs, but no DCX or MAP2-positive cells were detected. All data are reported as mean $\pm S D ; n=3$ independent experiments. Scale bars, $50 \mu \mathrm{m}$.

A
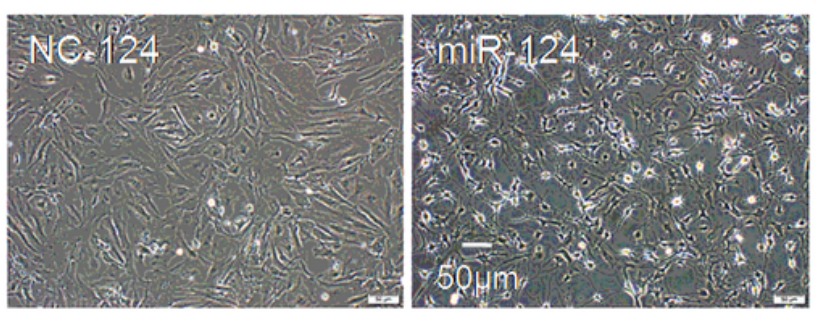

D

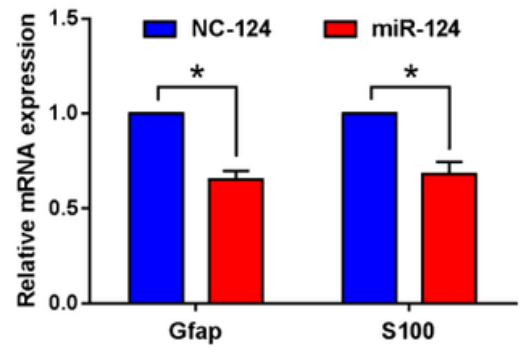

B

$E$

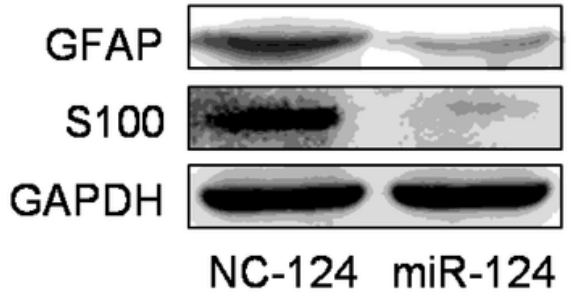

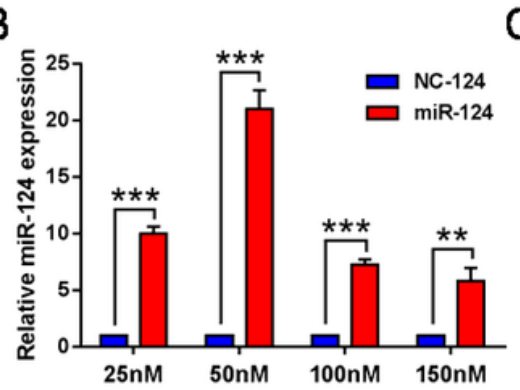

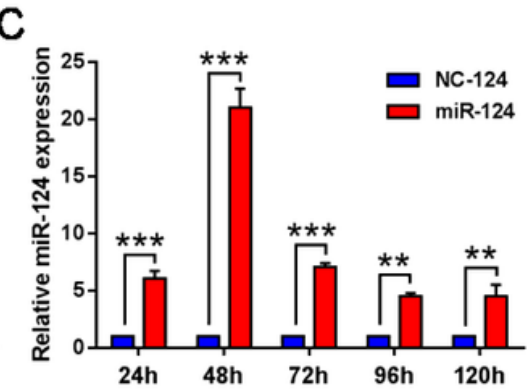

$\mathrm{F}$

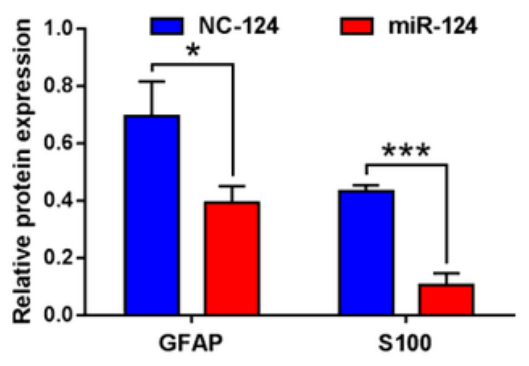




\section{Figure 2}

Overexpression of miR-124 in RAs attenuated astrocytic characteristics (A) Phase-contrast images of RAs transfected with miR-124 mimic (miR-124) or negative control (NC-124) at $48 \mathrm{~h}$. Notably, miR-124 group cells have smaller territories and more complex morphologies than NC-124 group cells. (B-C) qRT-PCR analysis after miR-124 overexpression at different concentrations and time points. The optimal transfection efficiency was obtained at $50 \mathrm{nM}$ for $48 \mathrm{~h}$. (D) Expression of astrocytic markers in miR124/NC-124 groups analysed using qRT-PCR. MiR-124 overexpression significantly downregulates Gfap and S100 mRNA expression in RAs. (E-F) Western blot (E) and densitometric quantitation (F) of GFAP and S100 in miR-124/NC-124 groups; signals were normalised to GAPDH. All data are reported as mean \pm SD; $n=3$ independent experiments $\left({ }^{*} P<0.05 ; * * P<0.01 ; * * * P<0.001\right)$. Scale bars, $50 \mu \mathrm{m}$.

A

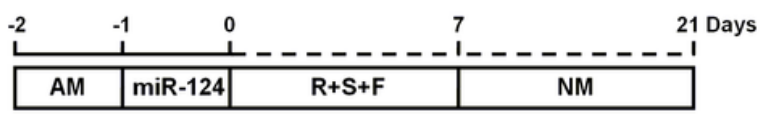

D

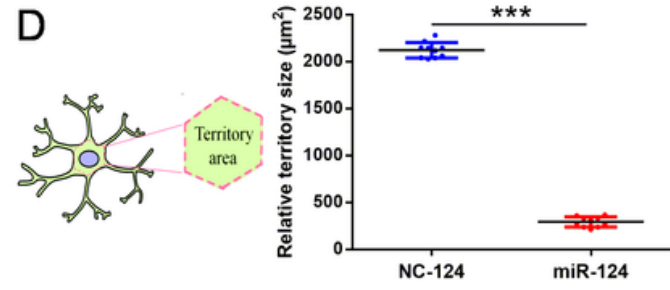

F

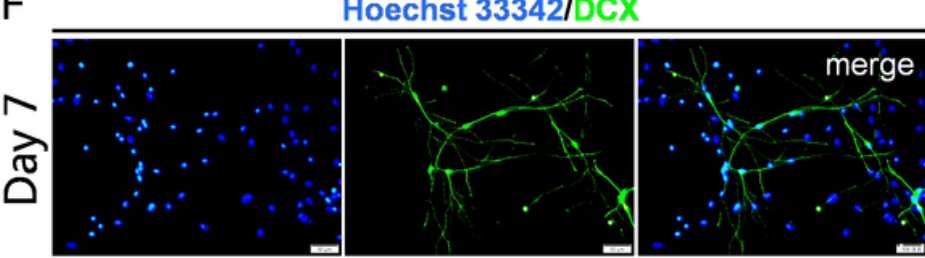

$\mathrm{H}$

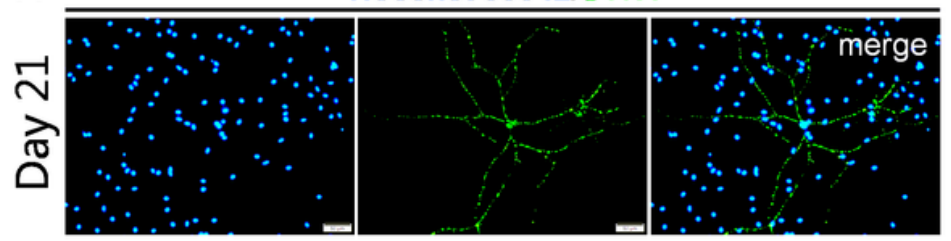

J

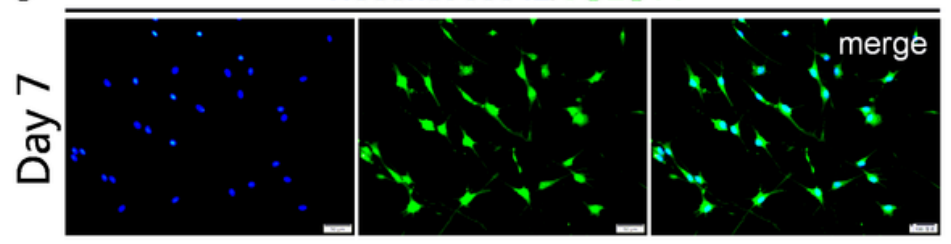

L

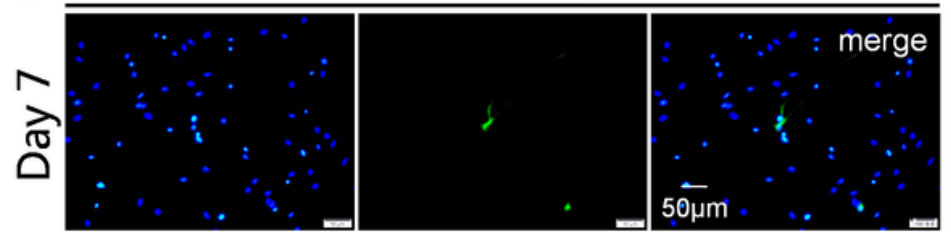

E
B

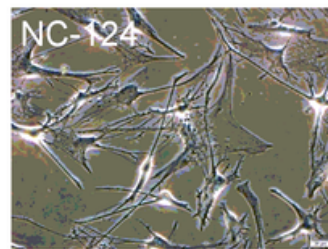

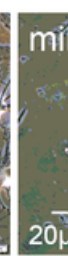

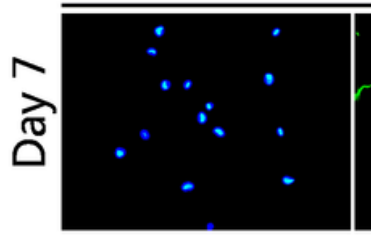

G
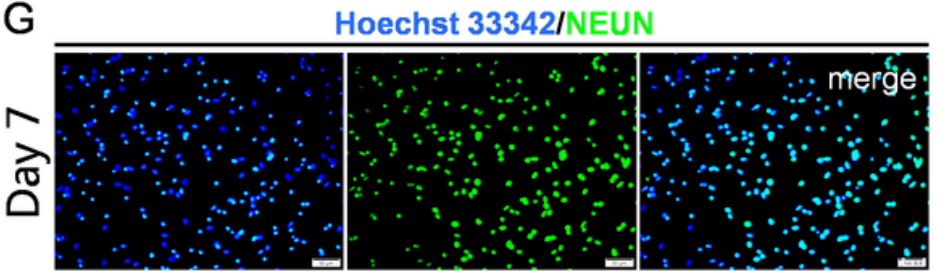

I

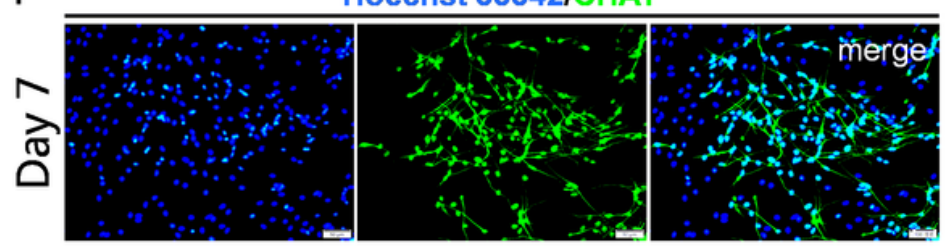

$\mathrm{K}$

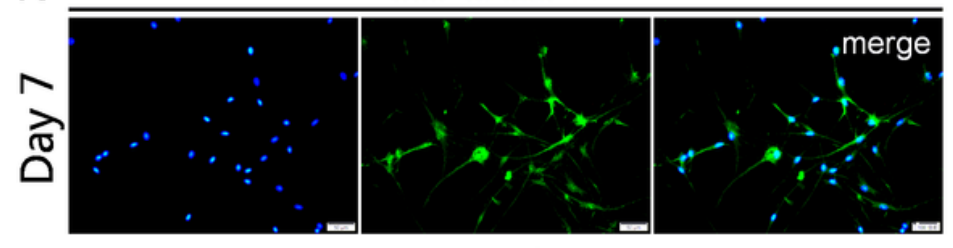

M

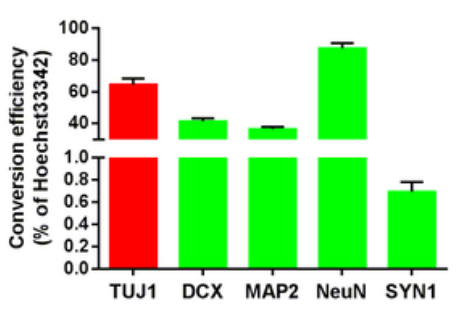

Hoechst 33342/MAP2/TUJ1
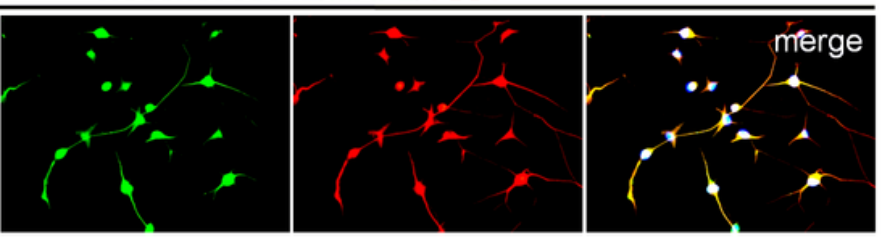

Hoechst 33342/NEUN

$\mathrm{N}$

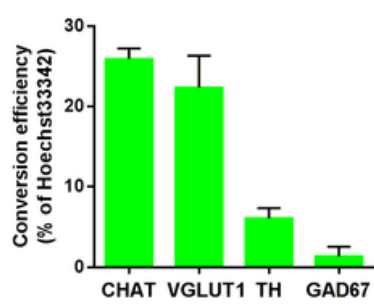

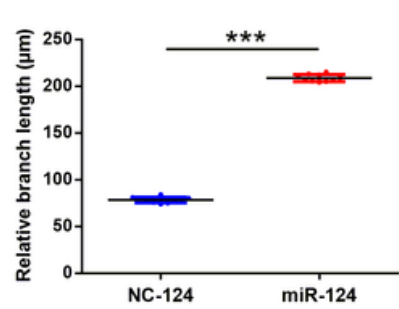




\section{Figure 3}

Conversion of RAs into iNs by miR-124 and small molecules under defined conditions (A) Schematic diagram showing the neuronal induction protocol. AM, RA conditioned medium; NM, neuron conditioned medium; R, ruxolitinib; S, SB203580; F, forskolin. (B) Phase-contrast images showing the change from astrocytic to neuronal morphology along the induction process. Bright field images of RAs induced with NC-124/miR-124 and small molecules at day 7. (C-D) Statistical analysis of the total branch length (C) and total territory size (D) of iNs (miR-124 group) and RAs (NC-124 group). Territory size is defined as the two-dimensional area that is delineated by the border of the cell body. $(\mathrm{E}-\mathrm{H})$ Immunostaining showing that iNs were positive for TUJ1, MAP2 (E), DCX (F), and NEUN (G) at day 7 and for SYN1 (H) at day 21. (IL) Immunostaining with different neuronal subtype markers reveals that iNs were mainly cholinergic (CHAT) (I), glutamatergic (VGLUT1) neurons (J), and rarely dopaminergic (TH) (K) or GABAergic (GAD67) $(\mathrm{L})$ neurons. (M-N) Quantification of neuronal conversion efficiencies $(M)$ and neuronal subtypes $(N)$ was performed. All data are reported as mean $\pm S D ; n \geq 3$ independent experiments ( $\left.{ }^{*} * * P<0.001\right)$. Scale bars, $20 \mu \mathrm{m}$ or $50 \mu \mathrm{m}$.

A
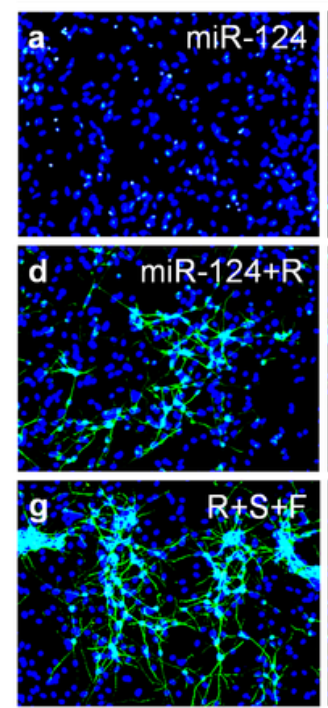

B
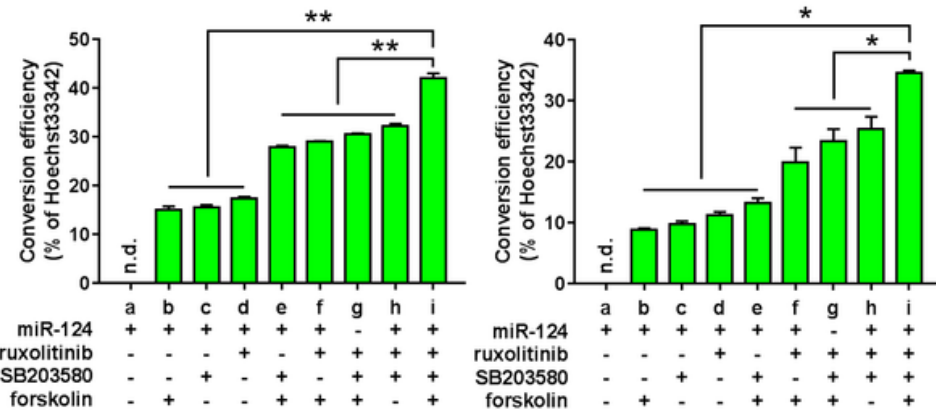

Hoechst 33342/DCX
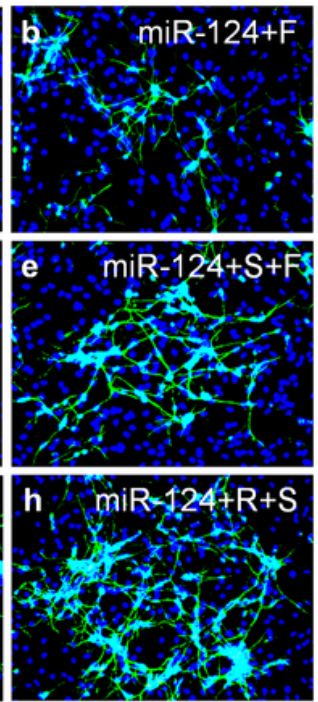

D
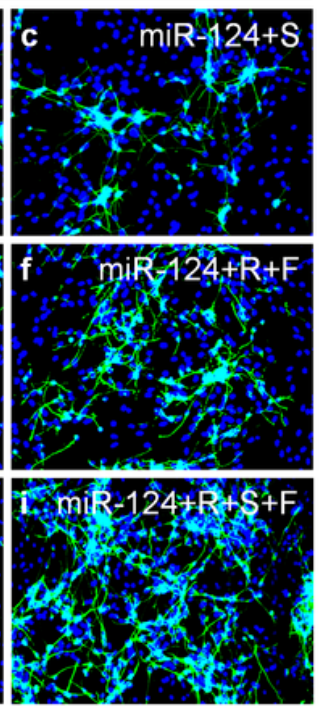

C
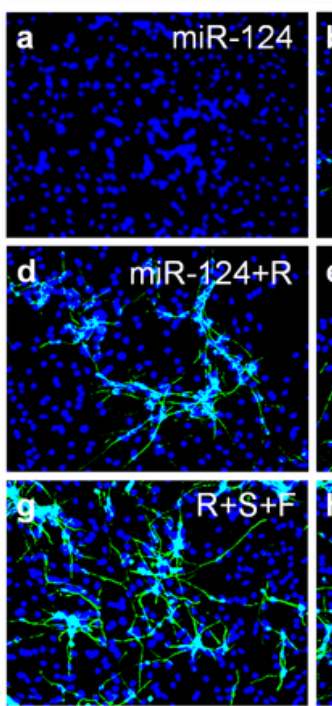

$\mathrm{E}$

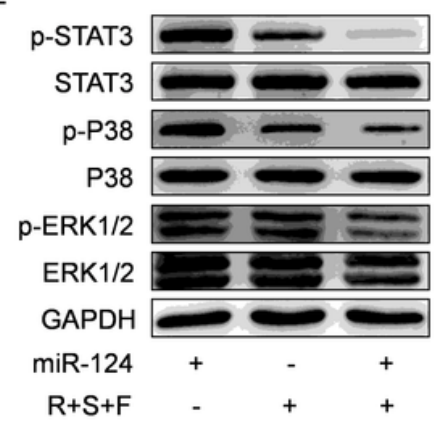

Hoechst 33342/MAP2
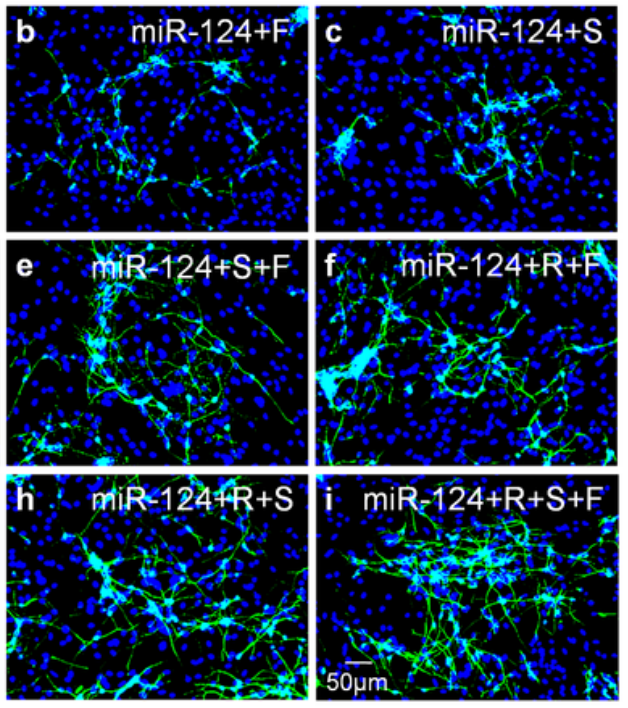

F

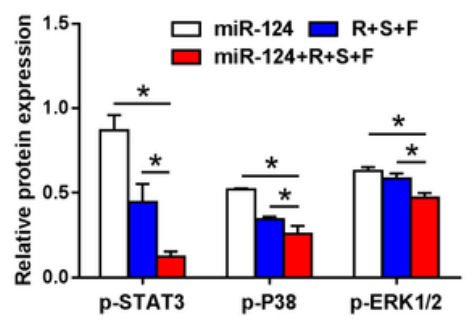

Figure 4

Regulation of RA-to-neuron conversion by miR 124 and the three small molecules (A-B) Representative profiles of immunostaining $(A)$ at day 7 and the percentage $(B)$ of DCX-positive cells after induction with 
different combinations of miR-124, ruxolitinib, SB203580, and forskolin. The conversion efficiency of miR-124 and the three small molecules treated group was higher than that of the other groups. (C-D) Representative profiles of immunostaining (C) at day 7 and the percentage (D) of MAP2-positive cells after induction with different combinations. The conversion efficiency of miR-124 and the three small molecules treated group was higher than that of the other groups. (E-F) Western blot (E) for measurement of phosphorylated STAT3 (p-STAT3), phosphorylated P38 MAPK (p-P38), phosphorylated ERK1/2 (pERK1/2), STAT3, P38, and ERK1/2 in RAs after induction with different combinations for $72 \mathrm{~h}$. GAPDH was used as a loading control. Quantification (F) was done by normalisation of p-STAT3 (p-P38 or pERK1/2) over total STAT3 (total P38 or total ERK1/2). All experiments were repeated thrice, and all data are reported as mean $\pm S D\left({ }^{*} P<0.05 ;{ }^{*} P<0.01\right)$. Scale bars, $50 \mu$ m. n.d., not detectable.

A

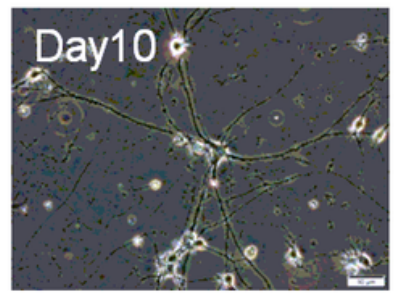

C

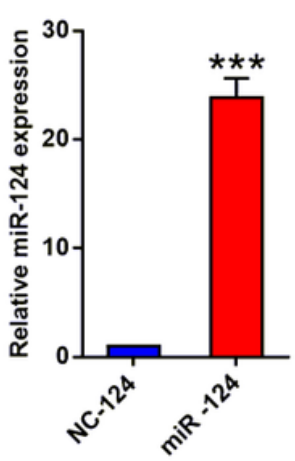

D

$\square$ iNs vs RAs
$\square$ iNs vs Rat neurons

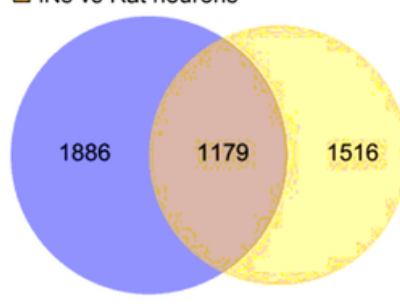

E

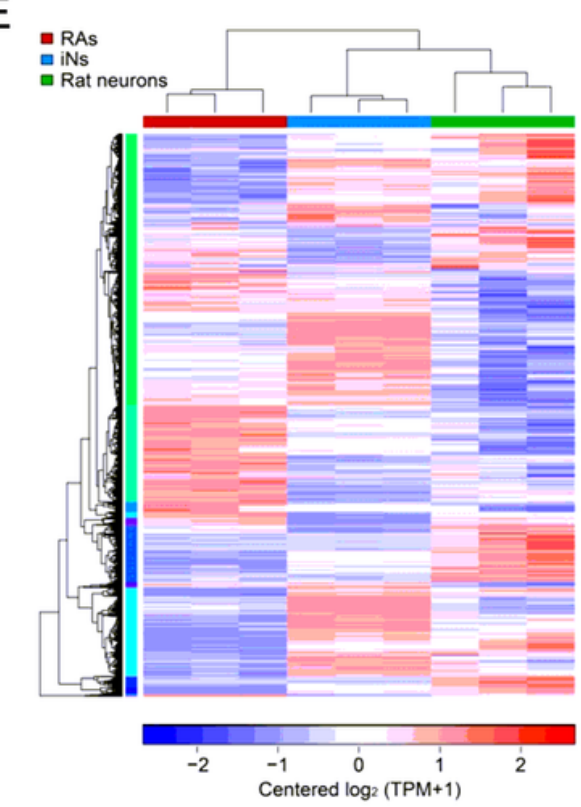

$\mathrm{F}$

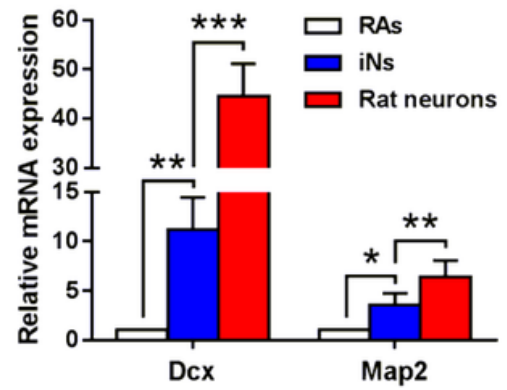

Figure 5

INs resemble rat cortical neurons (A) Bright field images of cultures showing typical neuronal morphology at day 10. (B) Immunostaining showing that the cultured cells expressed the neuronal markers TUJ1 and MAP2. (C) qRT-PCR analysis of miR-124 expression after induction at day 7. The overall miR-124 upregulation was mainly endogenous. (D) Venn diagram indicating that the number of sDEGs between iNs and RAs was more than that between iNs and rat neurons. (E) Heatmap illustration showing that the global expression profiles of iNs were more similar to that of rat neurons than of RAs. (F) qRT-PCR analysis of gene expression of neuronal markers between RAs, iNs, and rat neurons. The gene expression 
of iNs was closer to that of rat neurons. All data are reported as mean $\pm S D ; n=3$ independent experiments $\left({ }^{\star} P<0.05 ;{ }^{* *} P<0.01 ; * \star * P<0.001\right)$. Scale bars, $50 \mu \mathrm{m}$.

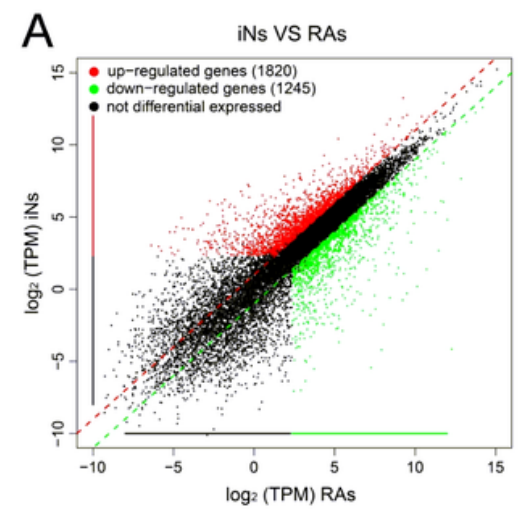

D

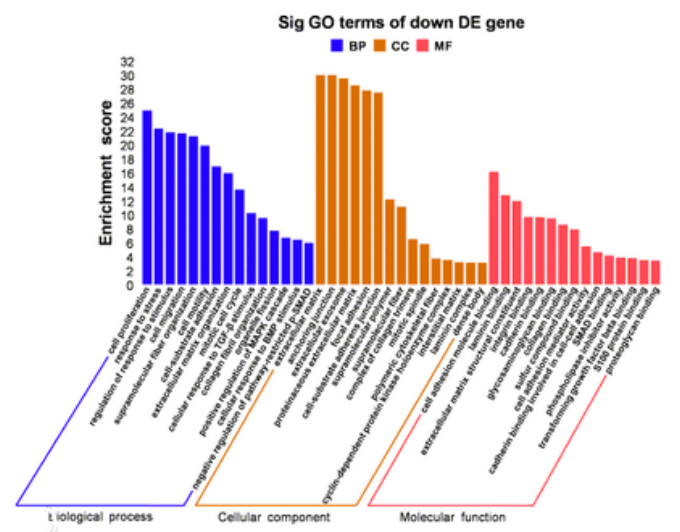

B

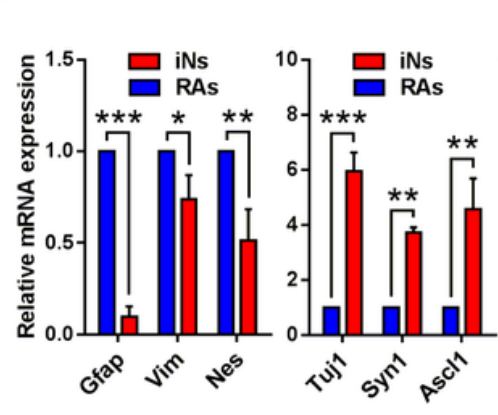

E

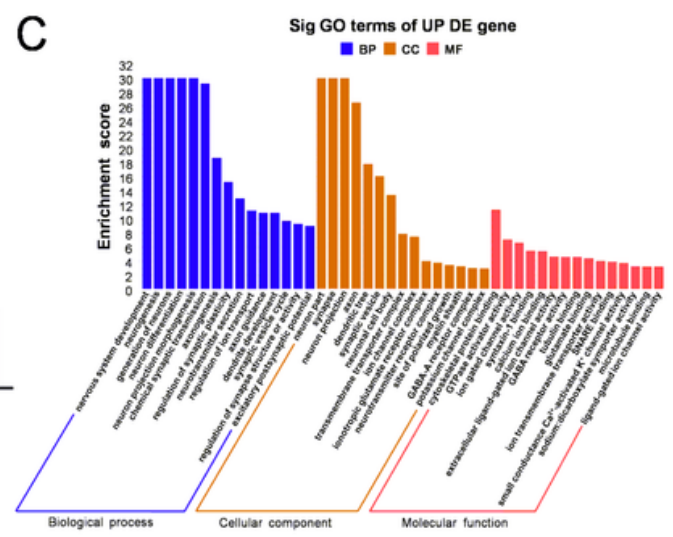

$\mathrm{F}$

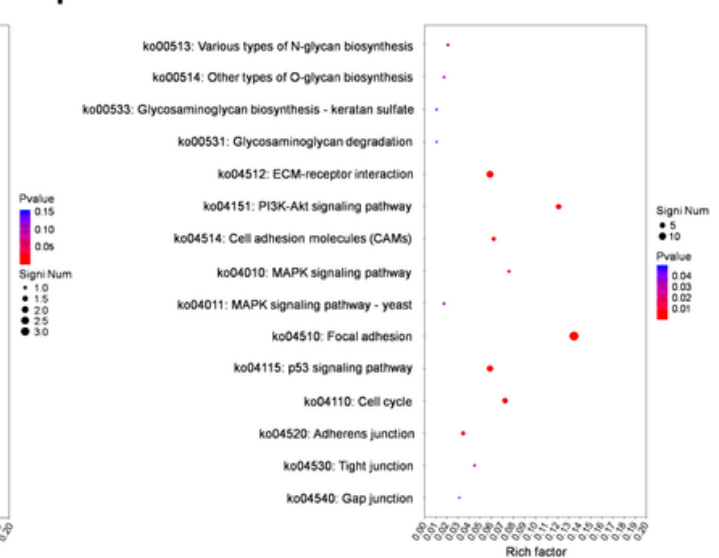

Figure 6

Comparison of transcriptome analysis between iNs and RAs (A) Scatter plots comparing gene expression levels between iNs (day 7) and RAs. Significantly upregulated genes are highlighted in red, significantly downregulated genes in green, and non-differential genes in black. Dashed line indicates TPM $\geq 5$ in at least one sample, |FoldChange| $>2$, and q-value $<0.05$. (B) qRT-PCR validation of the expression of representative RA- and neuron-enriched genes between RAs and iNs. Data are reported as mean \pm SD; $n=$ 3 independent experiments ( $\left.{ }^{*} P<0.05 ;{ }^{*} \mathrm{P}<0.01 ;{ }^{* \star *} \mathrm{P}<0.001\right)$. (C-D) GO enrichment analysis of upregulated/downregulated genes. The upregulated genes were significantly enriched in neuronal functions, and the downregulated genes were significantly involved in RA functions. (E-F) KEGG enrichment analysis of upregulated/downregulated genes. The upregulated genes were related to neuronal signals, and the downregulated genes were involved in RA signals. 
A

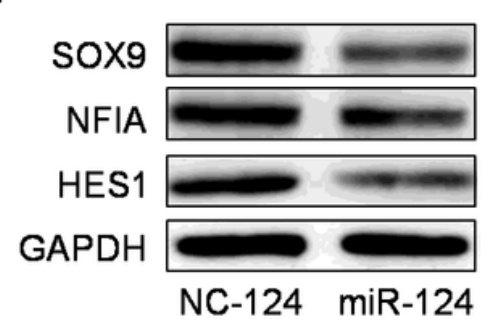

F

E

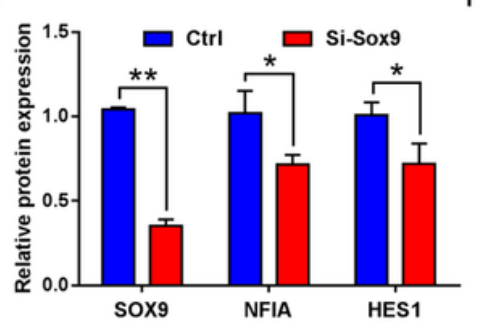

I

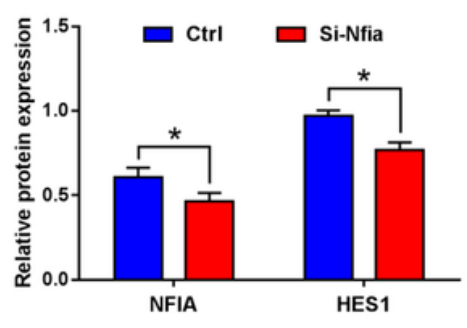

B

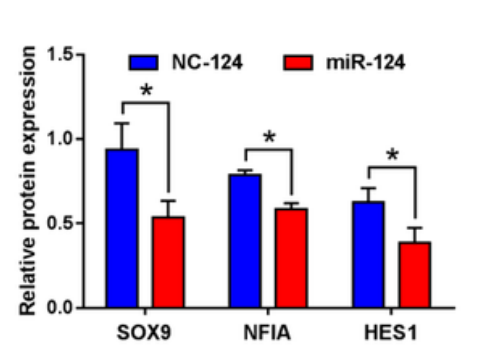

C

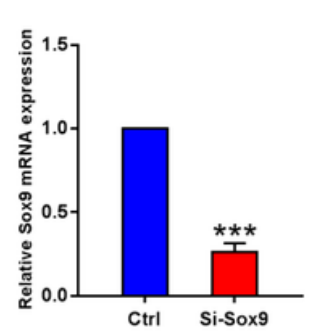

G

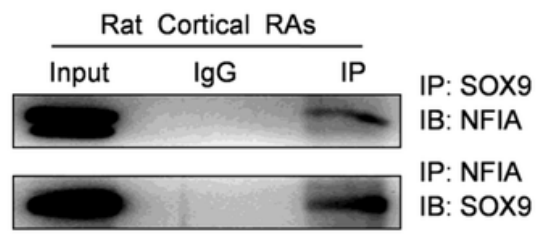

J

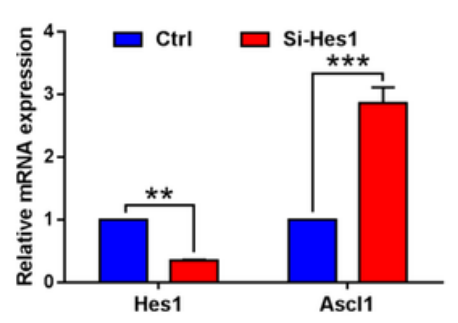

D

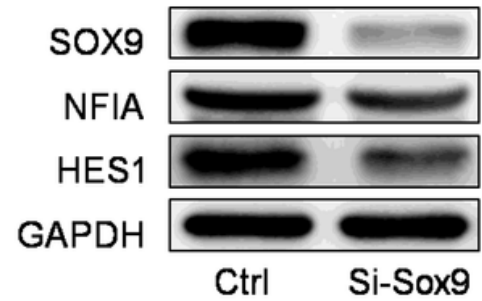

$\mathrm{H}$

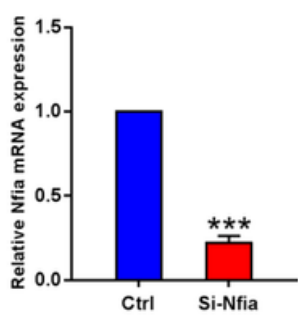

NFIA

HES1

GAPDH

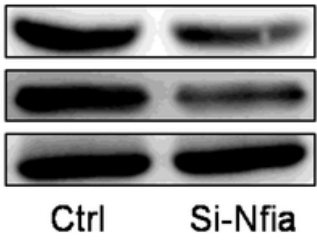

L

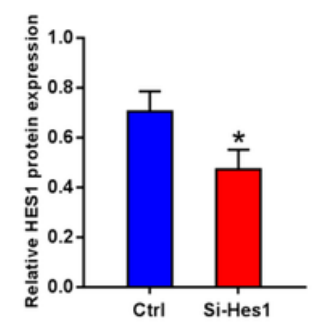

Figure 7

MiR-124 regulated HES1 expression via the SOX9-NFIA-HES1 axis in RAs (A-B) Western blot (A) and densitometric quantitation (B) for SOX9, NFIA, and HES1 expression in RAs after miR-124 overexpression at $96 \mathrm{~h}$. (C) The expression of Sox9 mRNA was assessed using qRT-PCR in RAs transfected with si-Sox9 or si-control (Ctrl) for 48 h. (D-E) The levels of SOX9, NFIA, and HES1 in si-Sox9 or Ctrl transfected RAs were determined by western blot (D) at 96 h, and quantification (E) was performed. (F) Coimmunoprecipitation shows that SOX9 and NFIA physically interacted in RAs. (G) mRNA expression of Nfia in si-Nfia or Ctrl transfected RAs analysed by qRT-PCR at $48 \mathrm{~h}$. (H-I) Expression of NFIA and HES1 was assessed by western blot in RAs transfected with si-Nfia or Ctrl for $96 \mathrm{~h}$. (J) Expression of Hes1 and Ascl1 at the mRNA level was analysed by qRT-PCR in RAs transfected with si-Hes1 or Ctrl for 48 h. (K-L) Western blot analysis (K) and quantitation ( $\mathrm{L}$ ) by densitometry for HES1 expression of RAs transfected with si-Hes1 or Ctrl at $96 \mathrm{~h}$ was performed. All protein expression levels were normalised to GAPDH. The experiments were repeated three times and all data are presented as mean $\pm S D$. $\left({ }^{*} P<0.05 ;{ }^{*} P<0.01\right.$; $\star \star * \mathrm{P}<0.001)$. 
A

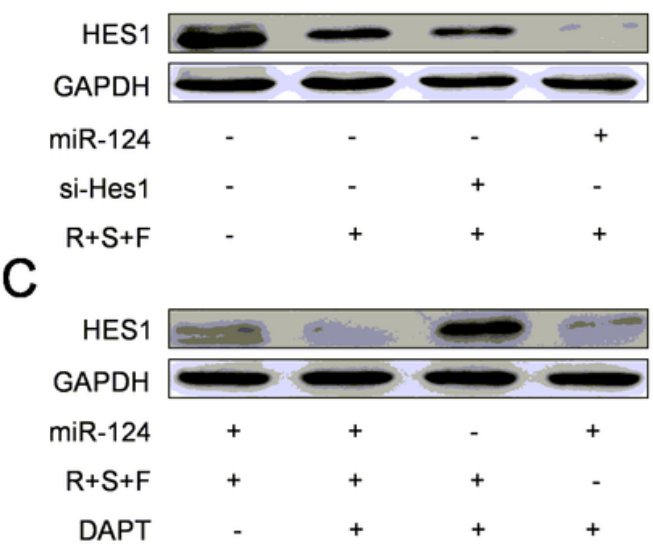

B

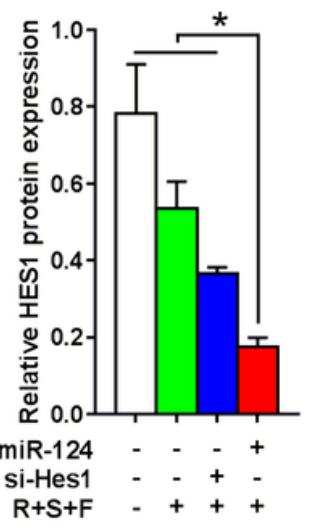

D

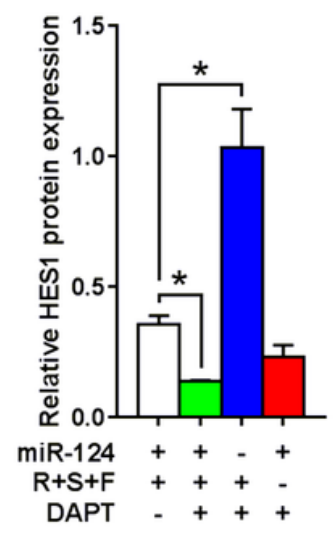

$\mathrm{F}$

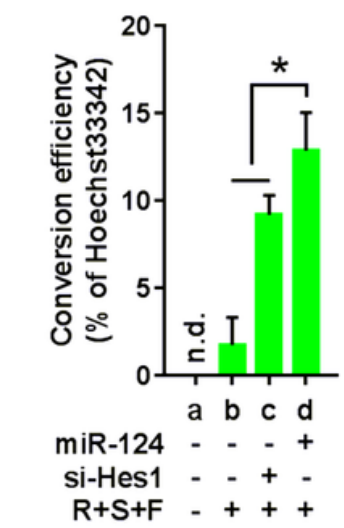

G

Hoechst 33342/DCX
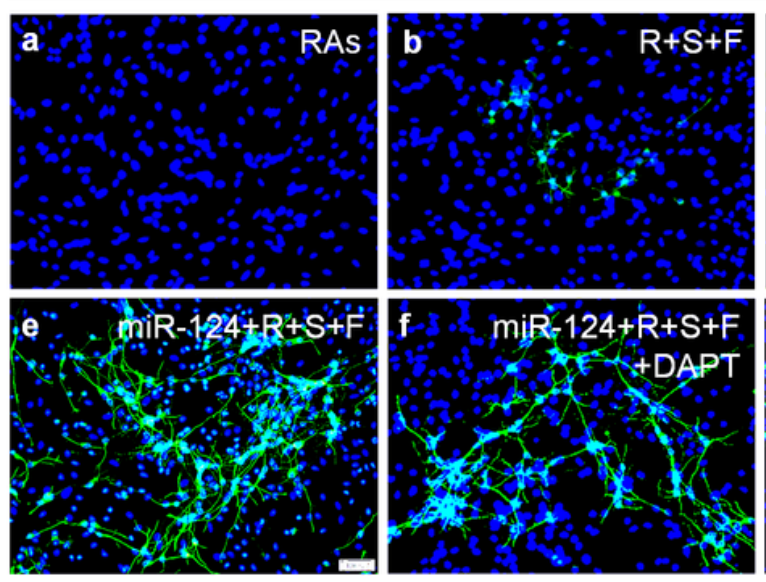
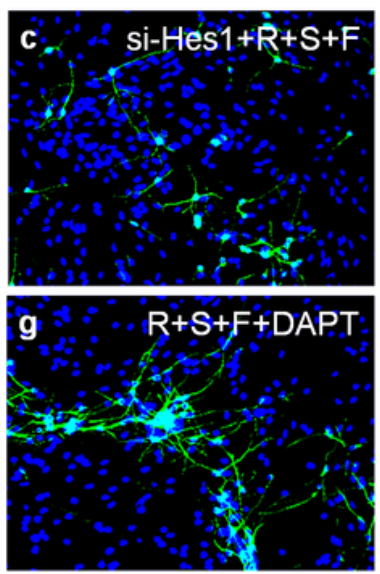
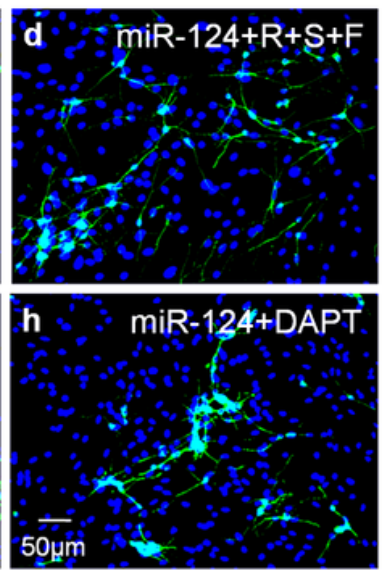

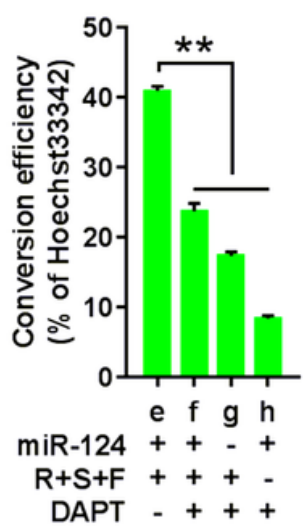

Figure 8

The RA-to-neuron conversion co-regulated by miR-124 and small molecules was associated with HES1 expression (A-B) Western blot (A) and densitometric quantitation (B) of HES1 expression in RAs, RAs treated with small molecules for 3 days, RAs treated with si-Hesl and small molecules for 3 days, and RAs treated with miR-124 and small molecules for 3 days. (C-D) Western blot (C) and quantitation (D) by densitometry for HES1 expression in RAs treated with miR-124 and small molecules for 7 days, RAs treated with miR-124, small molecules, and DAPT for 7 days, RAs treated with small molecules and DAPT for 7 days, and RAs treated with miR-124 and DAPT for 7 days. (E-G) Immunostaining (E) and the percentage (F-G) of DCX-positive cells in RAs, RAs after induction with miR-124/si-Hes1/DAPT and/or small molecules for $3 / 7$ days at different combinations. All protein expression levels were normalised to GAPDH. All experiments were repeated three times, and all data are presented as mean $\pm S D\left({ }^{*} P<0.05\right.$; $\star \star P<0.01)$. Scale bars, $50 \mu$ m. n.d., not detectable. 


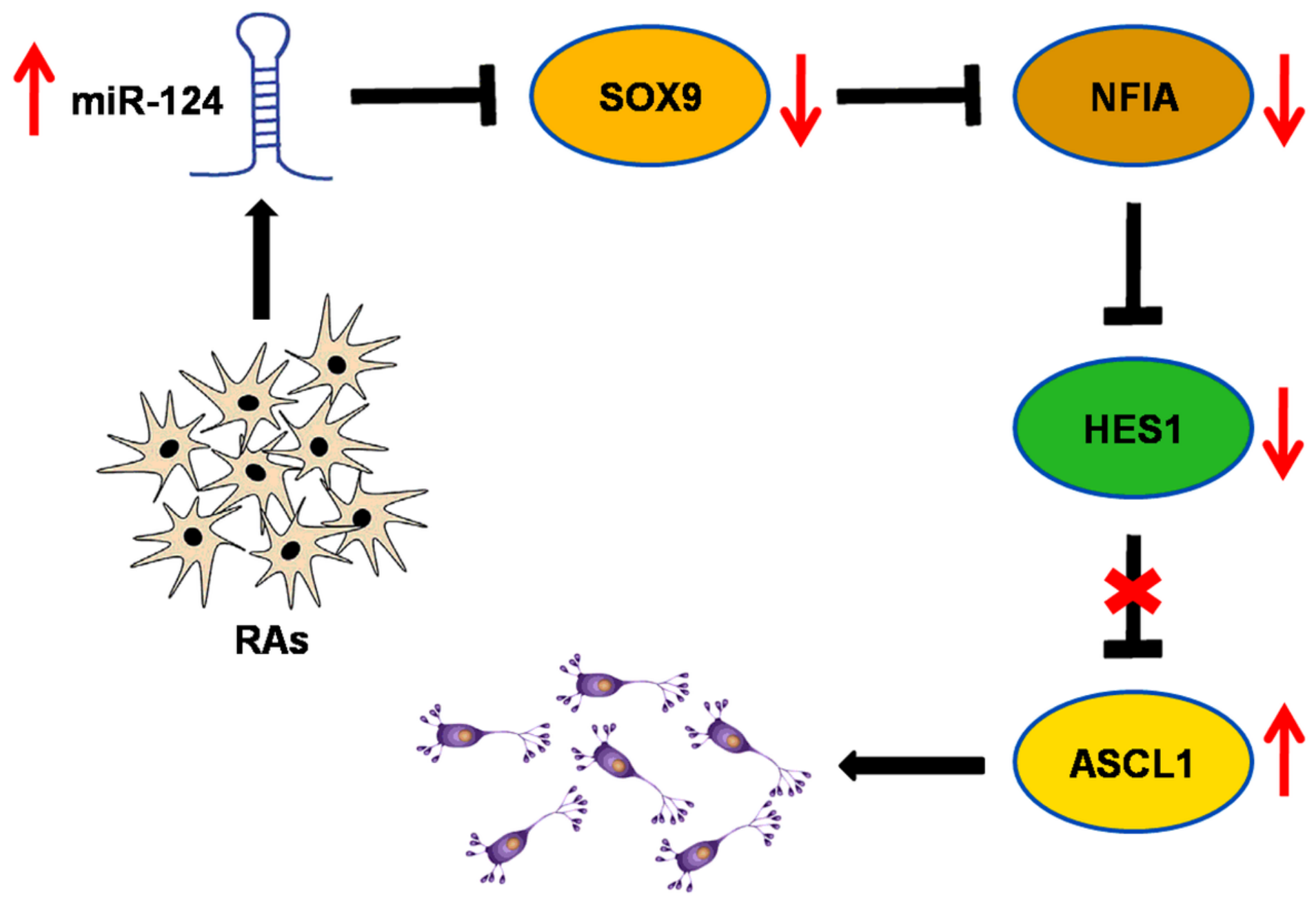

Neuronal differentiation

Figure 9

Proposed mechanistic model for miR-124-driven neuronal differentiation of RAs MiR-124 overexpression in RAs indirectly inhibited the expression of the NOTCH downstream target HES1 by targeting the SOX9NFIA-HES1 axis, thereby reducing the inhibitory effect of HES1 on ASCL1, and ultimately promoting the neuronal differentiation of RAs.

\section{Supplementary Files}

This is a list of supplementary files associated with this preprint. Click to download.

- Additionalfile120200815.doc 